\title{
Research Square \\ The complexity of the relationship between spontaneous brain activity and glucose metabolism
}

\section{Tommaso Volpi}

University of Padova https://orcid.org/0000-0002-5451-6710

\section{Erica Silvestri}

University of Padova https://orcid.org/0000-0002-1853-0777

Marco Aiello

IRCSS SDN, Napoli

\section{Maurizio Corbetta}

Department of Neuroscience, University of Padova

Alessandra Bertoldo ( $\nabla$ alessandra.bertoldo@unipd.it )

Padova Neuroscience Center

\section{Article}

Keywords: spontaneous brain activity, glucose metabolism, rs-fMRI

Posted Date: August 4th, 2021

DOl: https://doi.org/10.21203/rs.3.rs-728300/v1

License: (c) (1) This work is licensed under a Creative Commons Attribution 4.0 International License. Read Full License 

and glucose metabolism

6

7

8 Tommaso Volpi ${ }^{1,2 *}$, Erica Silvestri ${ }^{1,3}$, Marco Aiello ${ }^{4}$, Maurizio Corbetta ${ }^{1,2}$, Alessandra Bertoldo ${ }^{1,3 *}$ 9

${ }^{1}$ Padova Neuroscience Center, Padova, Italy

${ }^{2}$ Department of Neuroscience, University of Padova, Padova, Italy

15

${ }^{3}$ Department of Information Engineering, University of Padova, Italy

${ }^{4}$ IRCCS SDN, Naples, Italy and A.B. (bertoldo@dei.unipd.it) 
37 Brain glucose metabolism as assessed by $\left[{ }^{18} \mathrm{~F}\right] \mathrm{FDG}$ positron emission tomography (PET) is expected to be significantly related to resting-state functional MRI (rs-fMRI) activity and functional connectivity (FC), but the underlying coupling model is still incompletely understood. Employing simultaneous acquisitions, we related $\left[{ }^{18} \mathrm{~F}\right] \mathrm{FDG}$ standard uptake value ratio (SUVR) to 50 features

41 pertaining to rs-fMRI 1) signal, 2) hemodynamic response, 3) static and 4) time-varying FC, and 5) 42 phase synchronization. To assess which rs-fMRI variables better describe SUVR across regions, we 43 employed a hierarchical approach, identifying the model at population level, and then estimating it 44 on individual data. Multilevel modelling explained around $40 \%$ of the SUVR variance, with signal45 related features as the most relevant fMRI variables. When the model was used to characterize between-network variability of the SUVR-fMRI coupling, the ranking changed. We demonstrate that local activity and synchronization are the most important predictors of glucose metabolism, while large-scale FC properties gain importance within specific networks. 
Brain glucose consumption can be assessed in vivo by $\left[{ }^{18} \mathrm{~F}\right]$ fluorodeoxyglucose positron emission tomography ( $\left.\left[{ }^{18} \mathrm{~F}\right] \mathrm{FDG} \mathrm{PET}\right)$ acquisitions ${ }^{1}$, usually through semi-quantitative proxies like the standard uptake value ratio $(\mathrm{SUVR}){ }^{2}$. As evidenced by $\left[{ }^{18} \mathrm{~F}\right] \mathrm{FDG}$ studies, glucose metabolism displays significant regional variability. The reasons behind this heterogeneity in glucose expense, however, remain largely unexplained. Crucially, most of the remarkable metabolic budget of the brain, $\sim 25 \%$ of energy in the face of only $2 \%$ of body weight, is spent during rest ${ }^{3,4}$. This 'dark energy' of the brain ${ }^{5}$ with unclear functional meaning is expected to be mainly employed for maintaining resting potentials and subthreshold synaptic transmission ${ }^{6}$, since most of the energy budget of a neuron is utilized at the level of the synapses, rather than in the neuron's body ${ }^{7}$.

The regional differences in brain metabolism are thus likely to be explained by variability in spontaneous activity, which has been extensively explored with blood-oxygen-level-dependent (BOLD) resting-state functional magnetic resonance imaging (rs-fMRI) ${ }^{8,9}$. In addition to spontaneous activity, the functional relationships between activity patterns of different brain regions may relate to glucose consumption as well ${ }^{10}$. To this purpose, rs-fMRI can be used to derive the so-called "functional connectivity" (FC), i.e., the statistical relation between BOLD signal fluctuations in different brain regions; this approach has led to the identification of a functional architecture of resting-state networks (RSNs) that recapitulate clusters of regions activated for specific functions ${ }^{11,12}$. FC can be estimated in a static fashion ( $\mathrm{sFC}$ ), but also with time-varying approaches (tvFC), which interpret FC as non-stationary and changing across adjacent time windows ${ }^{13}$; notably, both might prove relevant to metabolic consumption.

Metrics derived from network science can then be used to characterize the topology of sFC and tvFC, with correlations between areas represented as 'edges' connecting 'nodes', and nodes described in terms of their centrality ('hubness'), number of connections, and so on ${ }^{14,15}$.

While rs-fMRI studies have provided a wide range of information on the properties of spontaneous activity, the physiological underpinnings of these results remain poorly understood, as the BOLD signal arises from a complex combination of cerebral blood volume (CBV), blood flow (CBF) and metabolic rate of oxygen $\left(\mathrm{CMRO}_{2}\right)^{17,18}$, and is indirectly and nonlinearly related to neuronal activity through the hemodynamic response function (HRF) ${ }^{19}$; importantly, it is also subjected to significant contamination from systemic modulations, both hemodynamic (heart rate variability, vasomotion etc.) and respiratory (e.g., respiratory volume variability $)^{20}$. Building upon the previous considerations, the relationship between the information provided by rsfMRI and $\left[{ }^{18} \mathrm{~F}\right] \mathrm{FDG}$ PET across brain regions needs to be thoroughly investigated with two main aims. 
89 First, to better understand the sources of regional metabolic variability. In particular, we might 90 wonder: 1) how much is the 'dark energy' of the brain related to local activity probed by the BOLD signal? 2) how much is instead related to local synchronization of the BOLD signal, i.e., a possible measure of neural population excitatory-inhibitory balance? 3) does inter-regional static synchrony play a more relevant role than more dynamic, time-varying interactions?

Second, the biological basis of the BOLD signal needs to be further characterized, an effort that would help turn rs-fMRI into a more specific biomarker; $\left[{ }^{18} \mathrm{~F}\right] \mathrm{FDG}$ PET, being tightly linked to neural activity $^{7}$, despite its typically low temporal resolution, can provide important insight. Some evidence on this relationship has started to emerge from sequential and simultaneous [ $\left.{ }^{18} \mathrm{~F}\right] \mathrm{FDG}$ PET/fMRI acquisitions ${ }^{21}$. In particular, the mean BOLD signal and the amplitude of its low frequency fluctuations (ALFF) have been found to be associated with $\left[{ }^{18}\right.$ F $]$ FDG SUVR across voxels ${ }^{22}$, with stronger correlations in specific brain regions ${ }^{23,24}$. With regard to the coupling between $\mathrm{FC}$ and metabolism, moderate associations between SUVR and global FC metrics were detected ${ }^{23,25}$, with stronger and more consistent correlations for the regional homogeneity (ReHo) of BOLD (up to Pearson's $r=0.8$ ), which is an index of local synchronization ${ }^{26,27}$. The topology of FC was also found to be important, with more central nodes having a stronger relationship between their FC and metabolic consumption ${ }^{23}$. In addition, the relationship between SUVR and local and global FC has been described as a power law or exponential model, especially in some specific networks ${ }^{25,28}$. The variability in the $\left[{ }^{18} \mathrm{~F}\right] \mathrm{FDG}-\mathrm{fMRI}$ coupling across networks has been highlighted in multiple previous studies $^{23,24}$, with demarcation between visual and default mode regions on one side, and frontoparietal regions on the other ${ }^{28}$. When the $\left[{ }^{18} \mathrm{~F}\right]$ FDG-fMRI coupling is assessed across subjects, however, much lower correlations between SUVR and rs-fMRI variables are detected in many studies ${ }^{23,26,27}$. In summary, somewhat inconsistent results emerge from the literature, with bivariate spatial correlations between $\left[{ }^{18} \mathrm{~F}\right] \mathrm{FDG}$ PET and a handful of BOLD-derived metrics (ALFF, ReHo, voxel-wise FC) ranging from 0 to 0.64 in explained variance $\left(\mathrm{R}^{2}\right)$, and substantial differences across brain regions and networks, as well as a low correlation across subjects even in simultaneous acquisitions ${ }^{23,27}$.

115 Notably, no study has ever attempted a multivariate integration of a wider range of rs-fMRI features, 116 as well as a multilevel prediction of SUVR both at the population level and at the subject/network 117 level.

118 We set out to fill these gaps in knowledge with a fully data-driven approach using simultaneously 119 acquired $\left[{ }^{18} \mathrm{~F}\right]$ FDG PET and rs-fMRI data from two separately published datasets of 26 subjects ${ }^{23,31}$. 120 After preliminary assessment of 50 rs-fMRI-derived variables, pooled into 5 categories, i.e., 1) signal, 121 2) HRF, 3) sFC, 4) tvFC, and 5) phase coherence (PC) ${ }^{29}$ (see Table 1 for the list of the features, their acronyms and a brief description) we set out to address the following questions. 
123 1. which is the strength of the bivariate association between these rs-fMRI features and SUVR across

124 the whole brain? And then, since regions with high vs. low metabolic consumption are expected to

125 have quite different structural and functional properties ${ }^{1,3,4}$, does this coupling change according to

126 the ranking of brain nodes based on SUVR?

127 2. is it possible to explain group level SUVR variance across regions by combining rs-fMRI features,

128 for the first time, into a multiple regression model? Is the group of selected features more populated

129 by local or large-scale brain network metrics, and does it account for between-subject variability

$130(\mathrm{BSV})^{30}$ ? Finally, which of the previously identified rs-fMRI features are more important to explain

131 SUVR when multilevel modelling is performed across RSNs, i.e., which is the between-network

132 variability (BNV) of the SUVR-fMRI association?

133

\section{Table 1 - Extracted rs-fMRI features and their categories}

135 Fifty fMRI-derived variables, divided according to the pool to which they belong: 1) signal, 2) 136 hemodynamic response function (HRF), 3) static functional connectivity (sFC), 4) time-varying 137 functional connectivity (tvFC), 5) phase coherence (PC). See Supplementary Methods for full 138 description of the features.

139

\begin{tabular}{|c|c|}
\hline Pools & rs-fMRI Variables \\
\hline \multirow{11}{*}{$\begin{array}{c}\widetilde{\sigma} \\
\stackrel{50}{0} \\
\ddot{0}\end{array}$} & med-BOLD: median of the BOLD time series \\
\hline & MAD-BOLD: median absolute deviation (MAD) of the BOLD time series \\
\hline & skew-BOLD: skewness of the BOLD time series \\
\hline & ApEn-BOLD: approximate entropy (ApEn) of the BOLD time series \\
\hline & rApEn-BOLD: range ApEn of the BOLD time series \\
\hline & $\begin{array}{l}\text { AR-BOLD: reflection coefficient of the first-order autoregressive AR(1) model fit to } \\
\text { BOLD time series }\end{array}$ \\
\hline & ALFF: amplitude of low frequency fluctuations (ALFF) of BOLD time series \\
\hline & ReHo: regional homogeneity of BOLD time series \\
\hline & MAD-ReHo: MAD of the time-varying ReHo (tvReHo) \\
\hline & CV-ReHo: CV\% of tvReHo \\
\hline & peaks-BOLD: number of BOLD pseudo-events \\
\hline \multirow{8}{*}{$\underline{\underline{a}}$} & peak-HRF: height of HRF peak \\
\hline & hrf-DEG: degree (DEG) of HRF correlation matrix \\
\hline & hrf-STR: strength (STR) of HRF correlation matrix \\
\hline & hrf-CC: clustering coefficient (CC) of HRF correlation matrix \\
\hline & hrf-BC: betweenness centrality (BC) of HRF correlation matrix \\
\hline & hrf-EC: eigenvector centrality (EC) of HRF correlation matrix \\
\hline & hrf-LE: local efficiency (LE) of HRF correlation matrix \\
\hline & hrf-GE: global efficiency (GE) of HRF correlation matrix \\
\hline \multirow{7}{*}{$\underset{\sigma}{U}$} & s-DEG: DEG of sFC \\
\hline & S-STR: STR of sFC \\
\hline & s-CC: $\mathrm{CC}$ of $\mathrm{sFC}$ \\
\hline & s-BC: $\mathrm{BC}$ of $\mathrm{sFC}$ \\
\hline & s-EC: $\mathrm{EC}$ of $\mathrm{sFC}$ \\
\hline & s-LE: LE of sFC \\
\hline & s-GE: GE of sFC \\
\hline
\end{tabular}




\begin{tabular}{|l|l|}
\hline mdiff-DEG: temporal median of the absolute value of $1^{\text {st }}$ order differentials (mdiff) \\
of DEG time series \\
mdiff-STR: mdiff of STR time series \\
mdiff-CC: mdiff of CC time series \\
\hline mdiff-BC: diff of BC time series \\
\hline mdiff-EC: mdiff of EC time series \\
\hline mdiff-LE: mdiff of LE time series \\
\hline mdiff-GE: mdiff of GE time series \\
\hline CV-DEG: coefficient of variation (CV\%) of DEG time series \\
\hline CV-STR: CV\% of STR time series \\
\hline CV-CC: CV\% of CC time series \\
\hline CV-BC: CV\% of BC time series \\
\hline CV-EC: CV\% of EC time series \\
\hline CV-LE: CV\% of LE time series \\
\hline CV-GE: CV\% of GE time series \\
\hline SampEn-DEG: sample entropy (SampEn) of DEG time series \\
\hline SampEn-STR: SampEn of STR time series \\
\hline SampEn-CC: SampEn of CC time series \\
\hline SampEn-BC: SampEn of BC time series \\
\hline SampEn-LE: SampEn of LE time series \\
\hline SampEn-GE: SampEn of GE time series \\
\hline med-LEig: median of the Leading Eigenvector (LEig)'s time series \\
\hline MAD-LEig: MAD of LEig time series \\
\hline CV-LEig: CV\% of LEig time series \\
\hline mdiff-LEig: mdiff of LEig time series \\
\hline
\end{tabular}

140

\section{Results}

\section{Feature extraction and preliminary evaluation of rs-fMRI variables}

The flowchart describing the preprocessing and preliminary analysis of the $\left[{ }^{18} \mathrm{~F}\right] \mathrm{FDG}$ PET and rsfMRI data is shown in Figure 1 (see the Methods section for details).

The $\left[{ }^{18} \mathrm{~F}\right]$ FDG PET variable of interest is the SUVR, which was extracted for every region of the Schaefer cortical atlas ${ }^{32}$ (200 parcels, supplemented by 18 subcortical regions ${ }^{33}$ ) in each subject, and will be considered as the dependent variable in every modelling approach from here onward.

The 50 rs-fMRI variables, extracted at the single-subject level and a priori subdivided into 5 pools, are reported in Table 1: the signal pool (1) contains features related to the basic statistics of the BOLD time series (median, variance, skewness), its complexity, its low-frequency fluctuations (ALFF), local coherence (ReHo) and high-amplitude events (peaks-BOLD); in the HRF pool (2), then, we placed the amplitude of the HRF peak, calculated using a blind deconvolution method ${ }^{34}$, and the HRF correlation structure across regions described by means of graph properties; the sFC pool (3) characterizes FC calculated across the entire fMRI scan with graph theory metrics; the tvFC pool (4) assesses graph metrics' temporal variability across sliding windows ${ }^{13}$; finally, the PC pool (5) characterizes FC as coherence of BOLD phase ${ }^{29}$. 
Figure 1 - Flowchart of rs-fMRI and $\left[{ }^{18}\right.$ F]FDG PET processing, feature extraction and analysis. Both rs-fMRI time series and $\left[{ }^{18} \mathrm{~F}\right]$ FDG SUVR data were parceled using the Schaefer cortical atlas (200 ROIs) and 18 subcortical ROIs. The parcel-wise rs-fMRI data were used to extract fifty features representative of five pools, i.e., 1) signal, 2) HRF, 3) sFC, 4) tvFC, 5) PC. The PET-fMRI coupling was investigated using bivariate correlation and multivariable multilevel modelling across subjects and across fMRI-based RSNs.

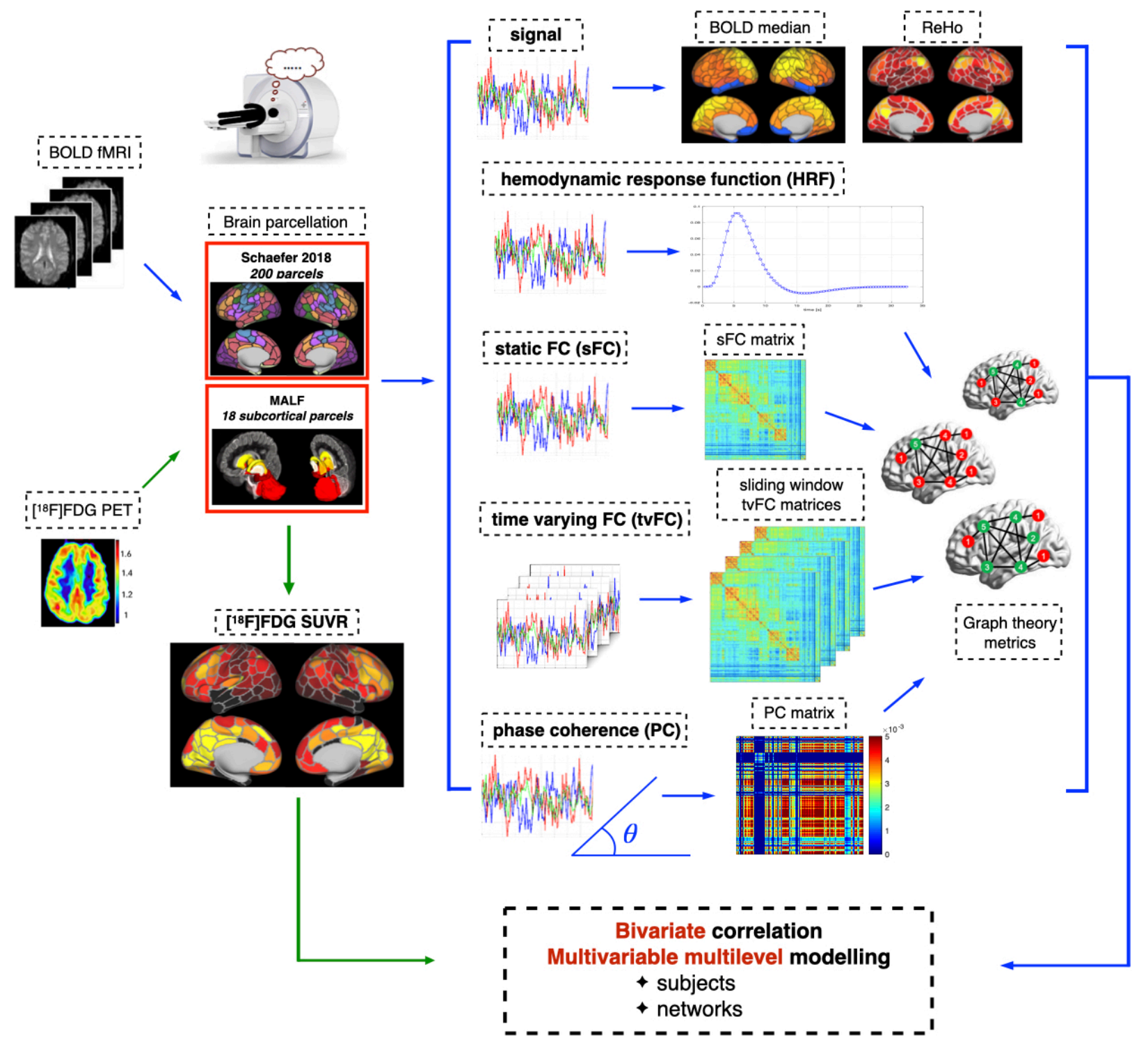


168 The Spearman's correlation matrix between the 50 rs-fMRI variables at group median level (i.e., by 169 taking the parcel-wise median value of each feature across subjects) was computed (Figure 2a), in order to assess the relationships between the extracted features and their degree of redundancy: the

171 clustering into 5 pools provided by a priori knowledge was fairly consistent with the observed 172 correlation structure, with signal, HRF and sFC features (upper block) being clearly distinguished 173 from tvFC features (lower block), which they are negatively correlated with, and PC variables 174 demonstrating lower correlation with the rest. However, it was also noticeable that strong correlations 175 between many variables were present, especially for the tvFC pool, and that a feature selection step 176 was going to be necessary to use these variables in a numerically sound multivariable model of 177 SUVR: the condition number $\kappa(X)$, which quantifies the level of correlation between predictors in a 178 multiple regression context (i.e., their multicollinearity), was high $(\kappa(X)=70.58)$, way beyond the 179 acceptability range ${ }^{35}$, and this is known to result in unstable and unreliable models (see Methods). SUVR vs. rs-fMRI: bivariate relationships Before moving to the multiple regression framework, we began by investigating bivariate associations between SUVR and the extracted rs-fMRI variables at the group level, in the so-called naïve average data approach (NAD), as done by many previous studies $23,25,26$; here, however, a much wider range of fMRI-derived variables was explored. Many significant spatial associations between SUVR and rs-fMRI features were detected across the 218 analyzed regions, as assessed through Spearman's rank correlation ( $\mathrm{p}=0.05$ significance level) with false discovery rate (FDR) multiple comparison correction $^{36}$. The correlation coefficients are reported in Figure $\mathbf{2 b}$. 

SUVR. The pattern of Spearman's correlations (FDR-corrected, non-significant values shown in white) among rs-fMRI features, assessed at the group level and divided according to the pool to which they have been assigned (1) signal, 2) HRF, 3) sFC, 4) tvFC, 5) PC), is shown in (a). The rs-fMRI features are tested for association with group median SUVR across 218 brain regions (b) via Spearman's correlations (significant values after FDR correction are indicated with an asterisk).

a

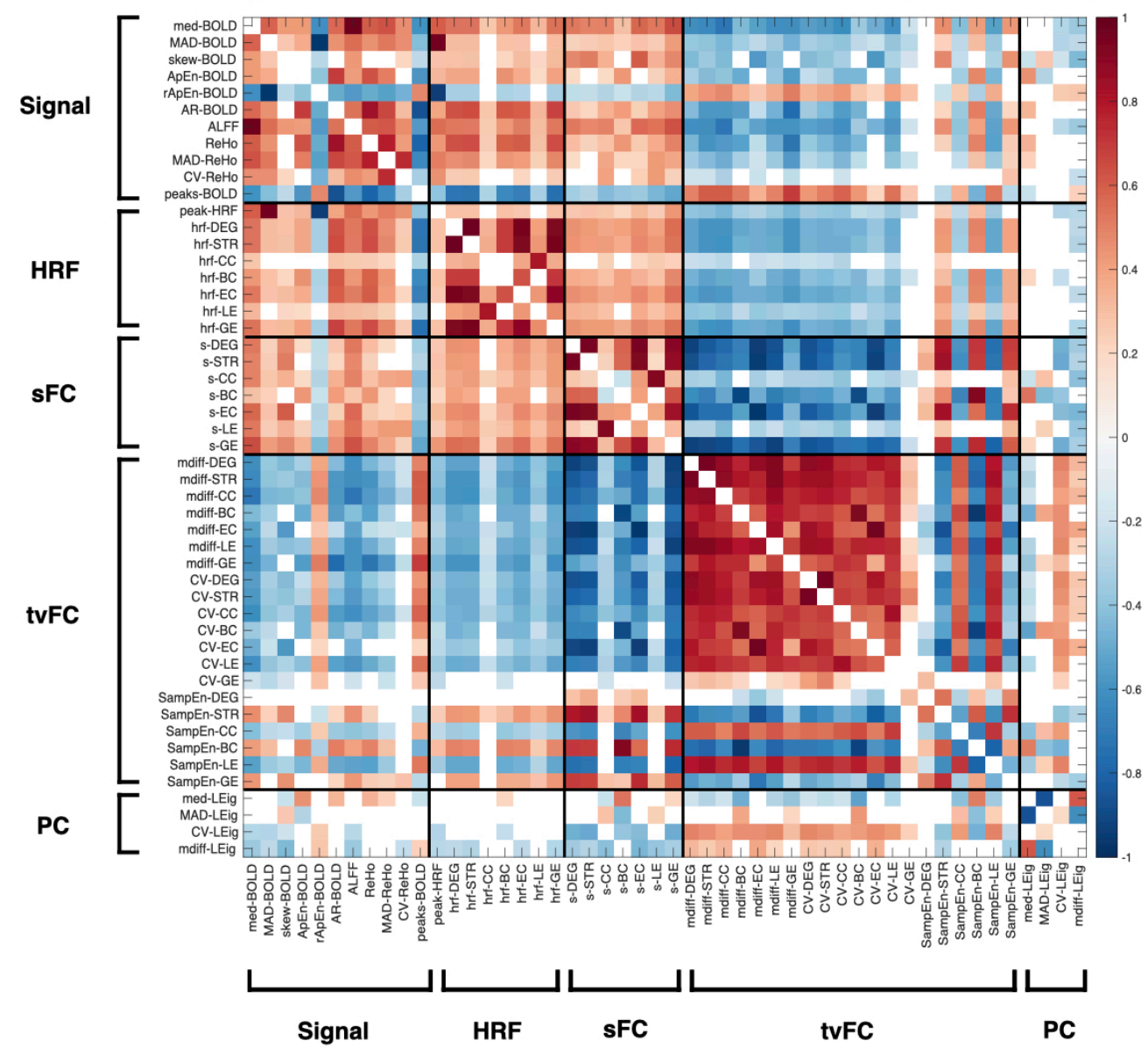

b

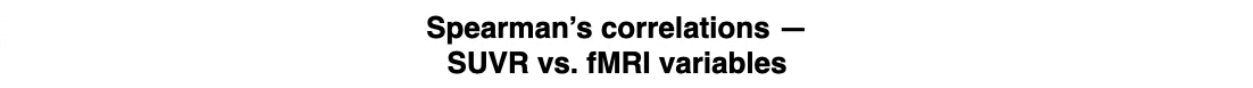

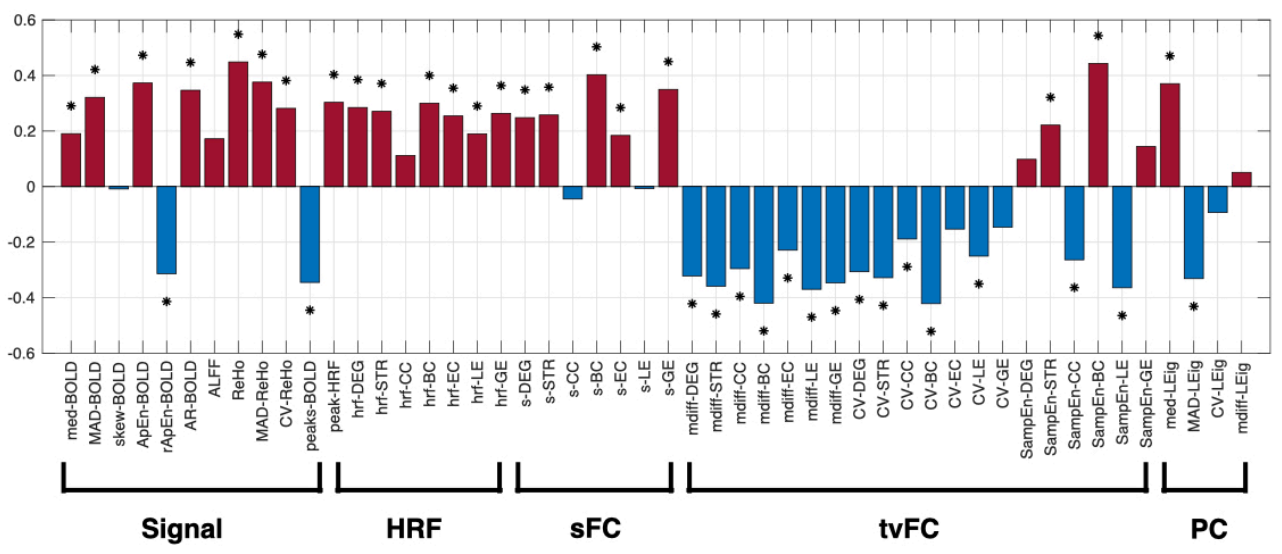


The strongest positive associations were with 1) ReHo $(\rho=0.45, \mathrm{p}<0.001), 2) \mathrm{s}-\mathrm{BC}(\rho=0.4, \mathrm{p}<$ $2010.001)$, and 3) SampEn-BC ( $\rho=0.44, \mathrm{p}<0.001)$, i.e., respectively 1) a measure of local synchronization of BOLD, 2) a sFC graph metric, betweenness centrality (BC), which describes a node in terms of its global connections in a graph, and 3) a measure of temporal complexity of the $\mathrm{BC}$ time series. The strongest negative correlations were mdiff-BC $(\rho=-0.42, \mathrm{p}<0.001)$ and $\mathrm{CV}$ $\mathrm{BC}(\rho=-0.42, \mathrm{p}<0.001)$ in the tvFC pool, both measures of temporal variability of BC. In general, it can be noted that positive associations emerged for the majority of the signal-based, $\mathrm{HRF}$ and sFC-related features, while tvFC metrics displayed a consistent and never previously reported negative association with SUVR (Figure 2b). Notable exceptions amongst signal-based features are rApEn-BOLD $(\rho=-0.31, \mathrm{p}<0.001)$, a measure of BOLD signal complexity, and peaksBOLD $(\rho=-0.34, \mathrm{p}<0.001)$, which quantifies the number of signal peaks exceeding one standard deviation from the baseline: both exhibited negative relationships with SUVR. Amongst tvFC features, SampEn-BC ( $\rho=0.44, \mathrm{p}<0.001)$ shows a strong positive coupling with SUVR, in contrast to the behavior of the other tvFC metrics. Interestingly, the dynamics of local synchronization, i.e., MAD-ReHo and CV-ReHo, displays a positive association with SUVR as well. Overall, it can be noted that the detected spatial correlations were at best moderate.

SUVR-fMRI associations are strengthened in low SUVR nodes

As the relationship between $\left[{ }^{18} \mathrm{~F}\right] \mathrm{FDG}$ PET and rs-fMRI could be spatially heterogeneous, Spearman's correlations were also re-evaluated across groups of nodes selected according to linearly increasing percentiles of the SUVR distribution, i.e., by progressively retaining the highest SUVR values, from the $1^{\text {st }}$ up to the $85^{\text {th }}$ percentile, as well as linearly decreasing percentiles of the same distribution, i.e., by retaining the lowest SUVR values, from the $100^{\text {th }}$ to the $15^{\text {th }}$ (Supplementary Figure S1). The purpose of the analysis was to verify whether SUVR-fMRI associations would be strengthened in high SUVR nodes or, conversely, in low SUVR nodes, as SUVR provides a clear ranking of brain regions, that is expected to be related to crucial structural and functional properties, e.g., neuron-to-glia ratio, richness in neuroreceptors, excitatory-inhibitory activity ${ }^{1,3,4}$. Spearman's correlations ( $\mathrm{p}=0.05$ significance level, FDR-corrected) between parcel-wise SUVR and all 50 rs-fMRI features (rows) are shown in Figure 3a, for each of the threshold levels along the SUVR distribution (columns). Going towards nodes with high SUVR (right side of Figure 3a) does not lead to any relevant effect for most features (except for three measures): therefore, hardly any strengthening of SUVR-fMRI relationships is detected in high SUVR nodes. Interestingly, however, a marked increase in many of the bivariate associations can be observed by selecting nodes with low values of SUVR (left side of Figure 3a), with highly significant correlations even after FDR correction. 


\section{Figure 3 - The SUVR-fMRI correlation changes strongly in low SUVR nodes.}

236 Spearman's correlations (FDR-corrected, non-significant values shown in white) between SUVR and all fifty rs-fMRI features (y axis) across nodes selected according to linearly increasing ( $x$ axis - right) and decreasing ( $x$ axis - left) percentiles of SUVR (a). The dashed black line shows the percentile with maximum correlation across features (i.e., nodes in the $1^{\text {st }}-40^{\text {th }}$ percentile range). The histogram (b), on the right, highlights the chosen percentile in the SUVR distribution and the range of percentiles included in the correlation. The brain regions shown on the left, plotted on the cortical surface and subcortex, are the parcels over which correlations are assessed.

a
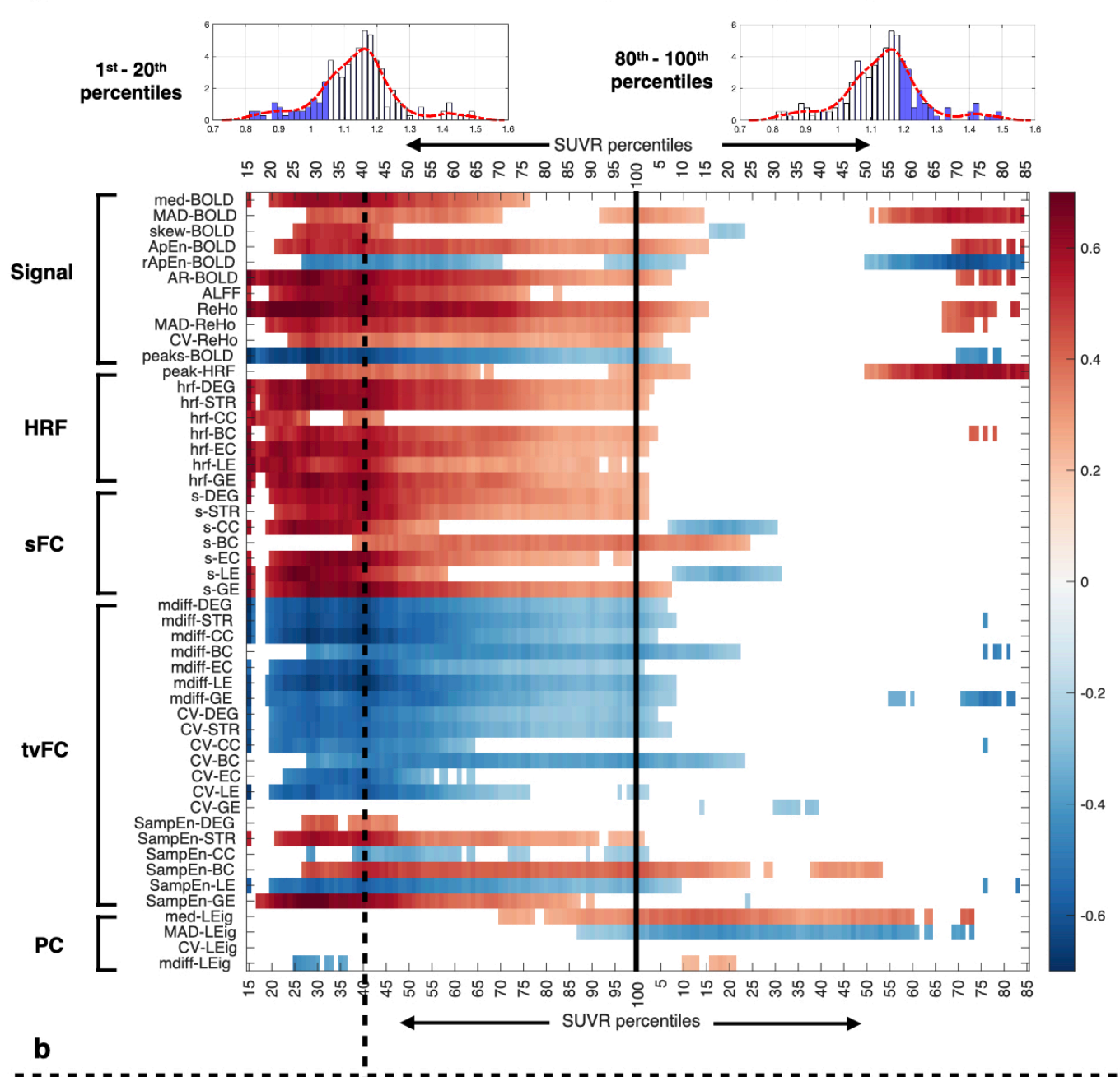

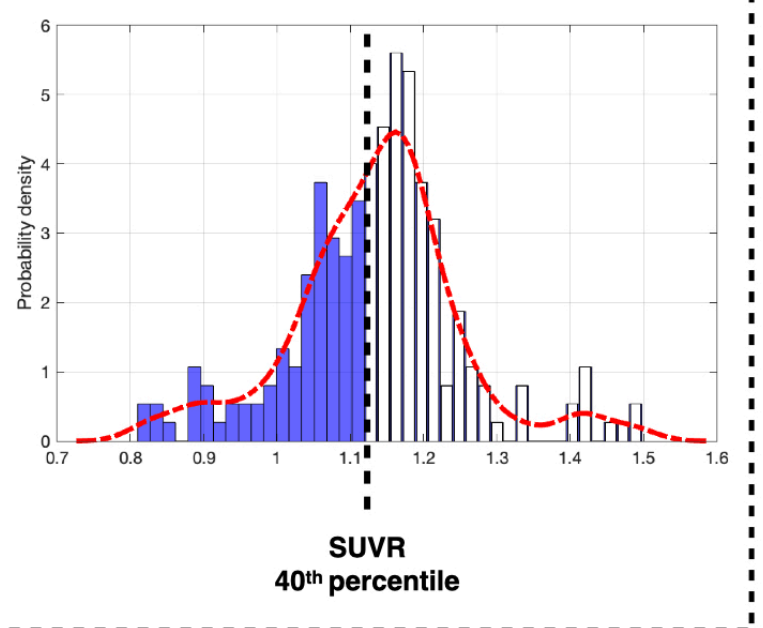


244 We then identified the percentile threshold corresponding to the highest total correlation value across 245 features: the spatial pattern of the 87 nodes below the $40^{\text {th }}$ percentile of the SUVR distribution is shown in Figure 3b. These parcels, where the SUVR-fMRI association is emphasized, mainly belong to temporal/limbic areas (including hippocampus), sensorimotor cortices, and subcortical regions, such as cerebellum and globus pallidus (Supplementary Figure S1).

This finding suggests the presence of nonlinear relationships between [ $\left.{ }^{18} \mathrm{~F}\right] \mathrm{FDG}$ SUVR and most rsfMRI features: tighter and more linear associations are present across a limited range of low SUVR nodes, with weaker coupling as SUVR gets higher. This nonlinear association was thus further characterized (see Supplementary Results), expanding on previous studies which were focused on specific networks and features ${ }^{25,28}$.

\section{SUVR vs. rs-fMRI: multivariable multilevel model}

We then set out to assess which combination of rs-fMRI features was better able to explain SUVR across brain regions with multiple regression and multilevel modelling, in a fully data-driven way. In multilevel modelling, the model structure is usually known, or selected at the lower level, i.e., at the individual level ${ }^{30}$. However, as significant BSV in the SUVR-fMRI association is expected, especially for the rs-fMRI features, we chose to identify the model predictors at the population level (NAD approach), thus exploiting the denoising properties of averaging. The model structure selected at the group median level was then used for multilevel modelling across subjects to characterize the BSV of the SUVR-fMRI association, as shown in (Figure 4, top), trying to fully capitalize on the fact that $\left[{ }^{18} \mathrm{~F}\right] \mathrm{FDG}$ and rs-fMRI data were acquired in the same subjects.

Maximum explanatory power of SUVR:

To assess the maximum explanatory power provided by the fMRI-derived features, we began by fitting an ordinary least squares (OLS) multiple regression model employing all the available features in a log-linear form (i.e., exploring the relationship between SUVR and the log-transformed rs-fMRI explanatory variables), to account for the detected nonlinearity (Supplementary Results): it can in fact be noted that the log-linear pairwise relationship between SUVR and each of the rs-fMRI features outperformed to the linear model in many occasions (62\%). From now we will call this log-linear model.

The OLS model had an $\mathrm{R}^{2}$ value of 0.62 : the maximum explanatory power thus reaches up to around $60 \%$ of the SUVR variance, without fully saturating the variance despite marked overparameterization (i.e., 50 rs-fMRI predictors). Due to the high number of predictors and the presence of multicollinearity, the precision of numerous parameter estimates (expressed as coefficients of variation, CVs\% ${ }^{37}$ was low, as expected (Supplementary Figure S3c). 
Figure 4 - Multilevel modelling approach for subjects and networks. The multivariable model structure defined at the population level for the SUVR-fMRI relationship was used in a hierarchical model. The multilevel approach was first applied to individual data with subjects $(S)$ as grouping variables $(S$, top $)$, then with networks $(N$, bottom $)$ : fixed effects $\theta_{S}$ and $\theta_{N}$ describe the population parameters, and random effects $\eta_{S i}$ and $\eta_{N j}$ describe how much each subject $i$ or network $j$ deviates from the population fixed effects for each of the selected parameters, i.e., the between-subject variability (BSV) and between-network variability (BNV) of the SUVR-fMRI model.

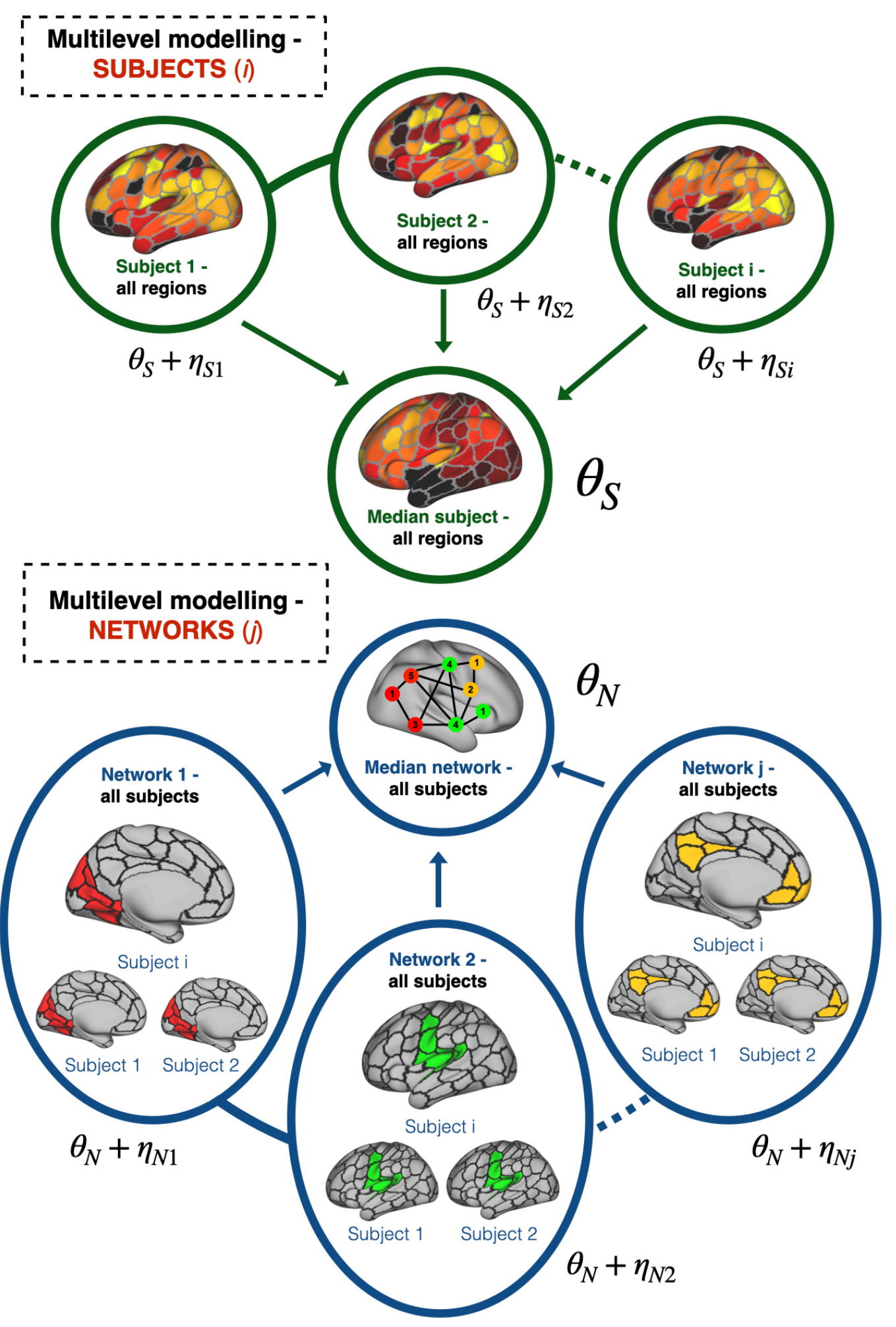


291 Multiple feature selection approaches (eleven methods) were then explored. The results of each and 292 the reason for the choice of the optimal approach are detailed in Supplementary Results.

293 The chosen feature selection process was performed in two stages. First, a sign-constrained non294 negative least squares (NNLS) estimator ${ }^{38}$ was employed; then, the NNLS estimates were refined with a second stage of feature selection with elastic net regression ${ }^{39}$. The reached solution was optimal in comparison with the other ten methods, in terms of both goodness of fit $\left(\mathrm{R}^{2}=0.411\right)$ and precision of the estimates (CVs\% $\mu \pm \sigma=66.73 \pm 17.79 \%$ ). The selected rs-fMRI predictors are: 1$)$ ApEnBOLD, 2) rApEn-BOLD, 3) ReHo, 4) CV-ReHo, 5) peaks-BOLD, 6) hrf-LE, 7) s-BC, 8) CV-BC, 9) med-LEig.

The first five predictors belong to the signal and local synchronization pool, while the other four to the remaining groups of rs-fMRI features, suggesting only an indirect relationship with the largescale network connectivity measures. Notably, most of the identified rs-fMRI predictors were chosen with high consistency across the employed feature selection methods, which highlights the robustness

Multivariable multilevel model across subjects:

The hierarchical modelling framework was then applied to the individual data using the identified predictors, to characterize the BSV of the SUVR-fMRI association. The log-linear model identified at the group level was re-estimated using a linear mixed-effect (LME) approach ${ }^{30}$. The fixed-effect $\left(\theta_{S}\right)$ parameter estimates, which represent the equivalent of the parameters estimated at the group median level, are reported in (Figure 5a) with their standard errors (SEs). To get an accurate ranking of the most relevant predictors in explaining SUVR, the estimated $\theta_{S}$ were ordered by their relative contribution to the model using dominance analysis (DA) ${ }^{40}$ (Figure 5b). In terms of general dominance (see Methods), at the top was ReHo (48\% of the total $\mathrm{R}^{2}$ ), followed by peaks-BOLD (19\%), CV-BC (11.74\%), CV-BC (10.50\%), s-BC (8.02\%), ApEn-BOLD (3.67\%), med-Leig (2.60\%), hrf-LE (1.47\%), rApEn-BOLD (0.02\%). Notably, the features belonging to the signal pool collectively accounted for $76.17 \%$ of the hierarchical model $\mathrm{R}^{2}$. The random effects $\left(\eta_{S i}\right)$ describe the deviation from the group value of the parameters for a specific subject $i$, i.e., how much the parameters of each subject $i$ are distant from the group-level estimates $\theta_{S}$ (Supplementary Figure S7b). In this case we found that the BSV in the SUVR-fMRI association is clearly non-negligible. In fact, the group-level $\theta_{S}$ estimates are very close to those obtained using the NAD approach, confirming the adequacy of the average approach in describing the relationship between the variables. However, as expected, the $\mathrm{R}^{2}$ of the overall model, i.e., considering BSV, was lower and equal to 0.245 , due to the capability of the multilevel mixed-effect approach to keep into account both 
325 between- and within-subject variability. The $\mathrm{R}^{2}$ values of the subject-level models are reported in 326 Supplementary Figure S7a, and they display high variability (from 0.05 to 0.45 ).

327 The median across subjects of the model's residuals $v_{S i}$, which highlight how well the SUVR of each 328 region is explained by the identified model, can be visualized in Figure 5c. Notably, high positive 329 values are present in posteromedial cortex (posterior cingulate cortex (PCC) in particular) and 330 subcortex (putamen): these areas identify nodes with high SUVR values which are not satisfactorily 331 explained by the available rs-fMRI features. Importantly, this deficiency in explanatory power is 332 highly consistent across subjects, as evidenced by the low variability (CVs\%) of the residuals in those 333 areas (Figure 5d). 
Figure 5 - Multivariable multilevel modelling of SUVR across subjects. The multivariable loglinear model predictors chosen at the group NAD level are shown. Parameter estimates and standard errors for the fixed effects $\theta_{S}$, which represent the parameters that best explain SUVR across regions at the group level (a). The relative importance weights produced by dominance analysis (DA), highlighting the proportion of the multivariable multilevel model $\mathrm{R}^{2}$ explained by each predictor (general dominance) (b). Across-subject median (c) and CVs\% (d) of weighted residuals $v_{S i}$ of the multilevel model, plotted on the brain cortex and subcortex.

a

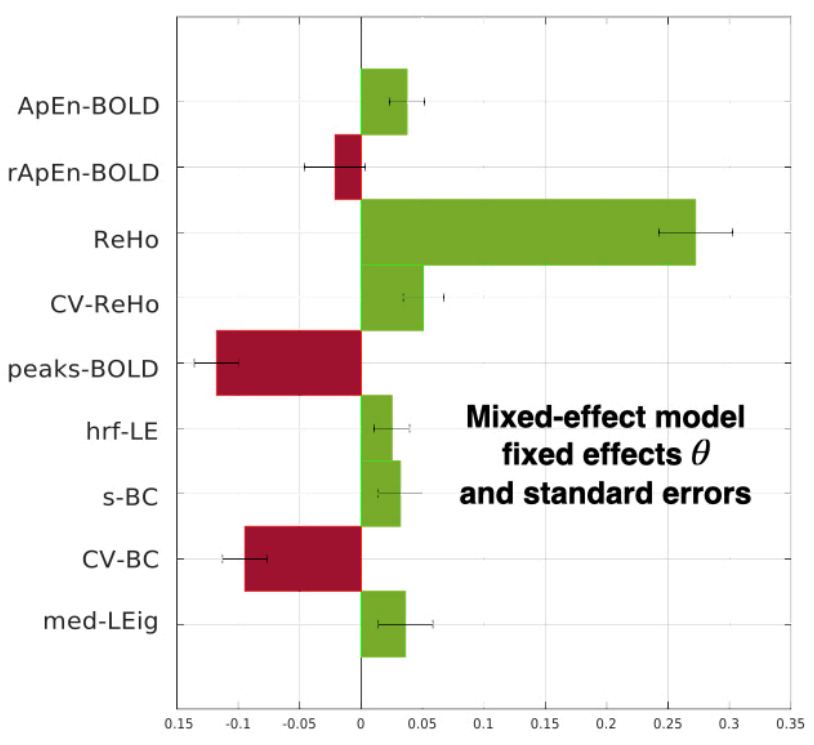

C

Median of residuals across subjects

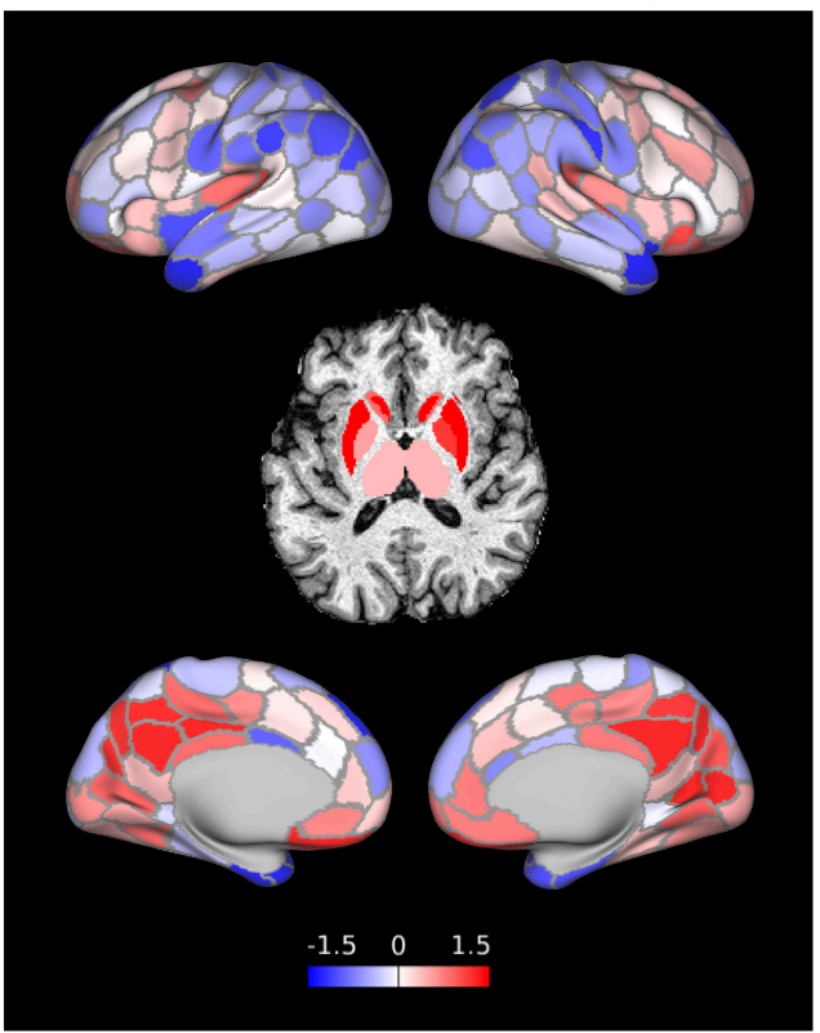

b

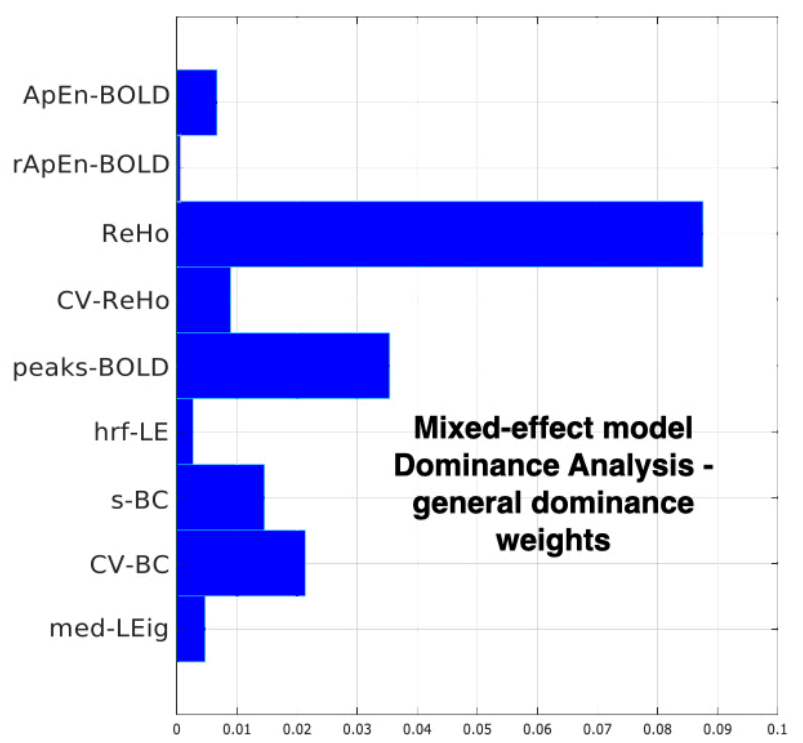

d CV\% of residuals across subjects

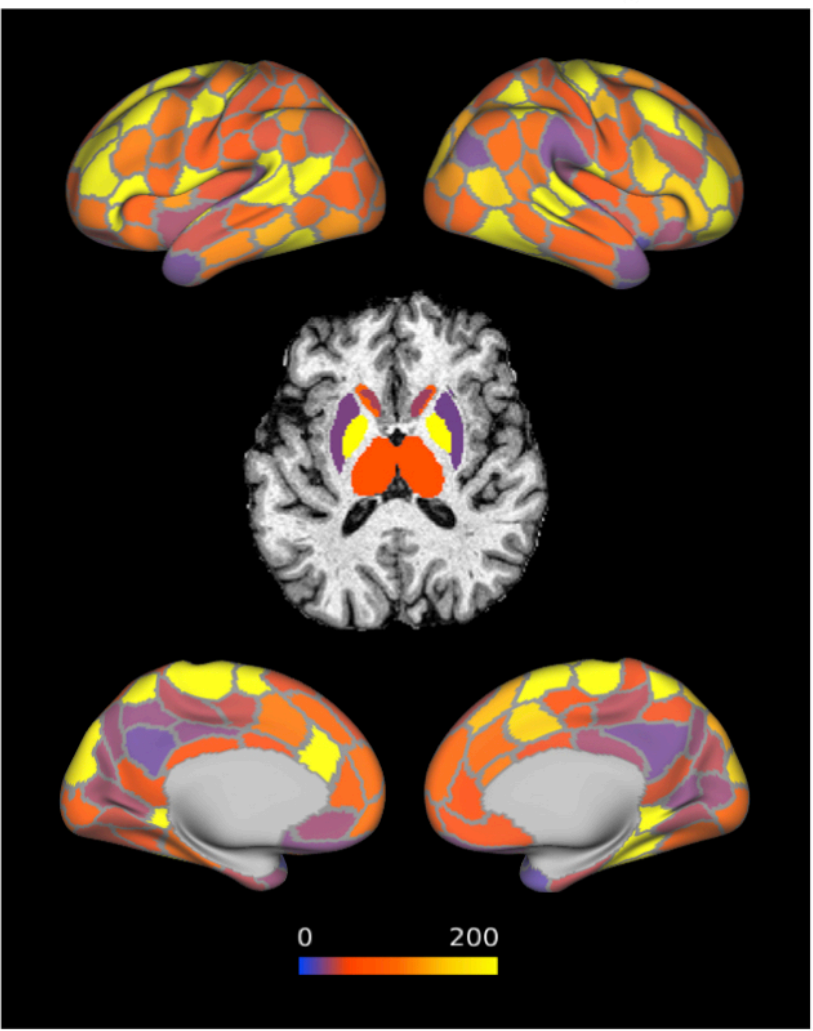


346 Finally, the log-linear model with the 9 selected rs-fMRI predictors was tested in its ability to describe 347 the expected network-level variability of the SUVR-fMRI association (BNV), i.e., by considering 348 only the parcels referring to specific brain networks. RSNs were here grouped according to the 349 Schaefer' functional atlas in its 17-RSN partition ${ }^{32}$, supplemented by 18 subcortical anatomical 350 regions $^{33}$. A multilevel modelling framework was again employed, but with RSNs as the grouping 351 factor for individual level data, instead of subjects (Figure 4, bottom).

352 The fixed effects $\theta_{N}$ and their SEs for the between-network model are reported in (Figure 6a). ReHo 353 and peaks-BOLD are still highly relevant parameters in describing the SUVR across networks, 354 together with ApEn-BOLD and CV-BC; rApEn-BOLD and hrf-LE, instead, lose importance, and 355 their fixed effect $\theta_{N}$ becomes irrelevant (with a SE range crossing the zero-line). To confirm the 356 ranking, DA was performed in this context as well: ReHo was still the most important predictor in 357 terms of general dominance (explaining $23.24 \%$ of the model's $\mathrm{R}^{2}$ ), followed by CV-BC (19.85\%), 358 peaks-BOLD (16.39\%), s-BC (15.19\%), ApEn-BOLD (11.46\%), med-LEig (9.65\%), CV-ReHo 359 (2.55\%), hrf-LE (1.80\%), rApEn-BOLD (0.13\%) (Figure 6b).

360 Notably, the $\mathrm{R}^{2}$ of model prediction considering network-wise estimates is markedly lower than when 361 subjects are used to cluster nodes. As shown in (Figure 6c), the single RSNs are highly heterogeneous 362 in terms of model $\mathrm{R}^{2}$, ranging from around 0 to 0.32 , with an overall prediction with $\mathrm{R}^{2}=0.147$. 
Figure 6 - Multilevel SUVR modelling across networks - parameter estimates and explained variance. The nine features chosen at the group level are shown. Parameter estimates and standard errors for the fixed effects $\theta_{N}$, which represent the parameters that best explain SUVR across regions in an average network (a). Relative importance weights produced by dominance analysis (DA) in terms of the proportion of the between-network model $\mathrm{R}^{2}$ explained by each predictor (general dominance) (b). Network-wise $\mathrm{R}^{2}$ values, representing the percentage of SUVR variance explained by the mixed-effect model at the network level, plotted on the cortical surface and subcortex (c).
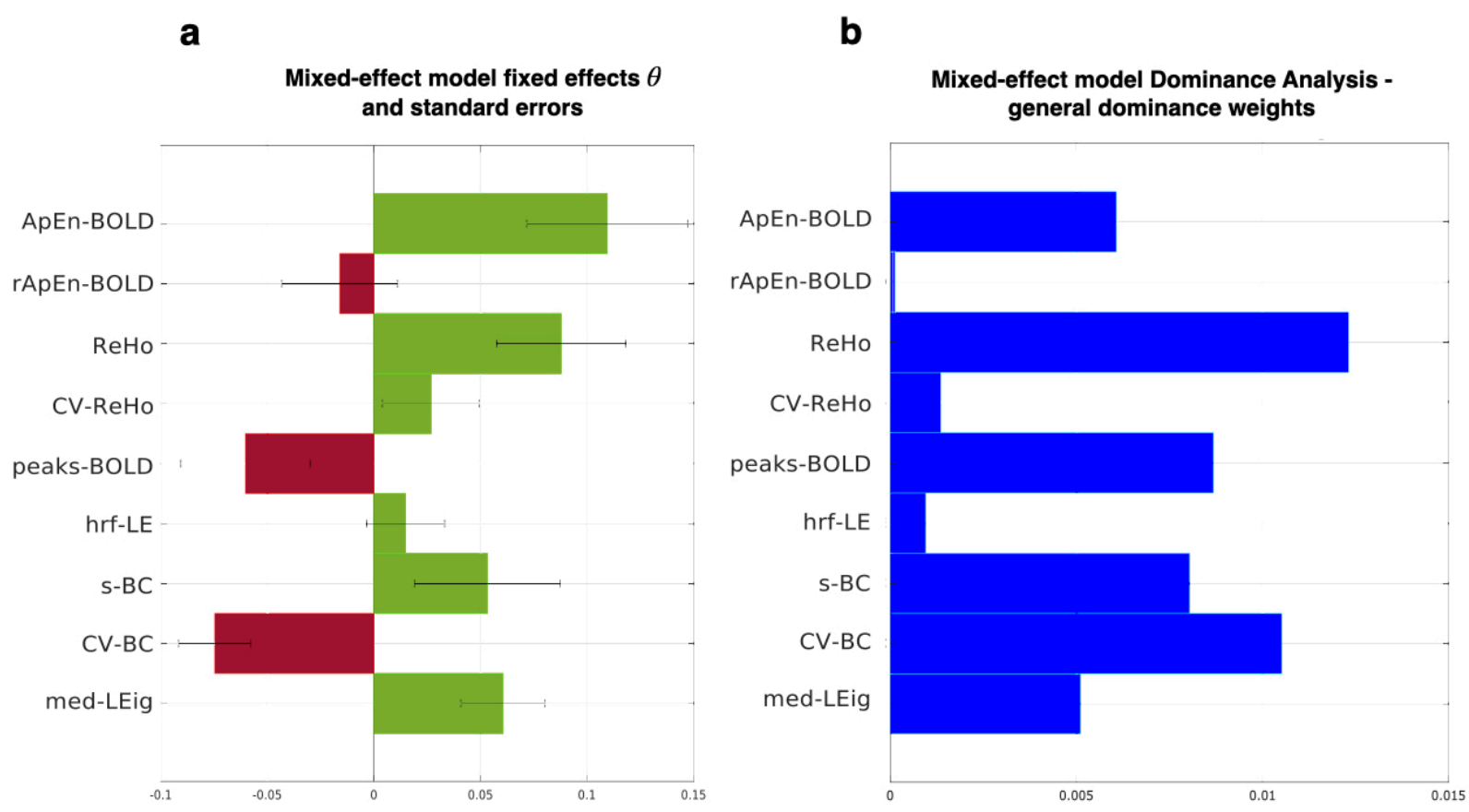

C

Network-wise model $\mathbf{R}^{2}$

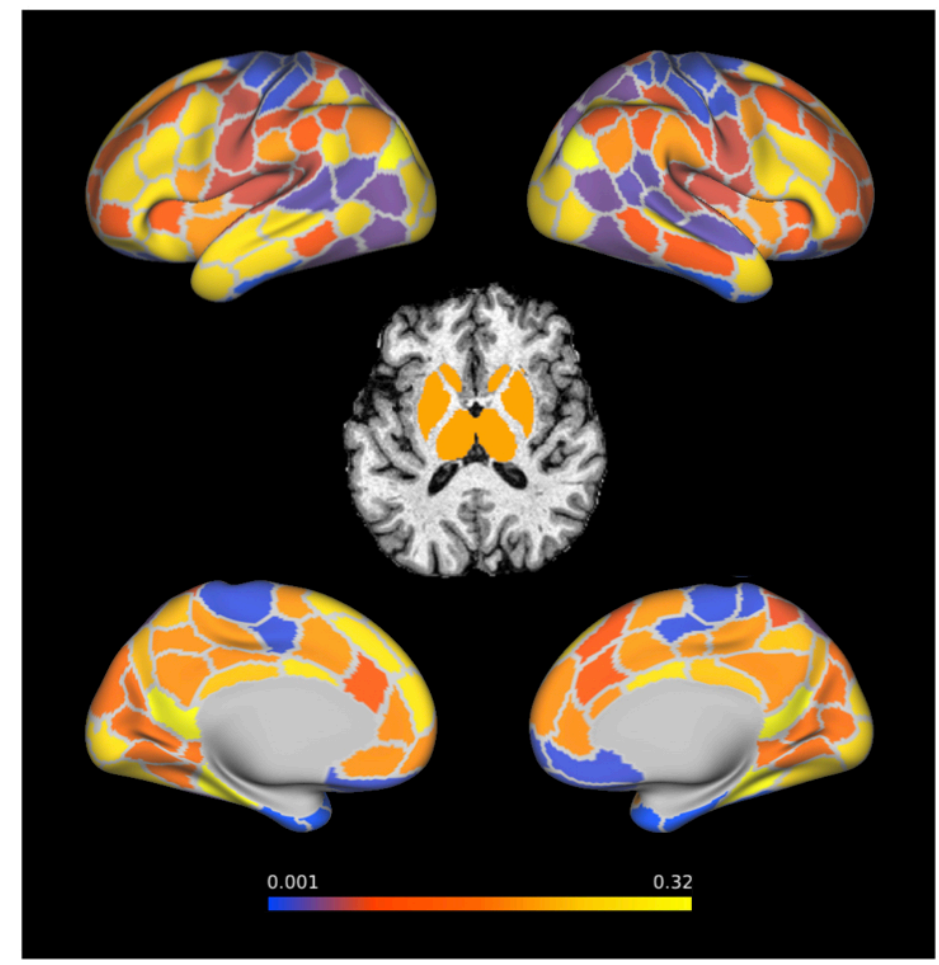


373 The BNV of the SUVR-fMRI association is measured by the random effects $\eta_{N j}$ for each network, 374 with some RSNs displaying significant distance from the model estimates $\theta_{N}$ of the "average375 network". To better assess this variability, the nine rs-fMRI predictors' parameter estimates $\psi_{N j}$ (i.e., 376 sum of fixed effects $\theta_{N}$ and random effects $\eta_{N j}$ for every network $j$ ) were plotted (Figure 7a). We 377 can observe that most predictors included in the multivariable model display heterogeneity across 378 networks in their relationship with SUVR, with either positive or negative associations depending on 379 the specific RSN, which cannot be captured by the average situation described by the fixed effects $380 \theta_{N}$ of (Figure 6a). Some predictors show notably consistent spatial patterns, and therefore, to assess 381 their similarity, the correlation between their random effects was evaluated across networks (Figure 382 7b). Notably, although uncorrected for multiple comparisons, significant correlations $(\mathrm{p}<0.05)$ can 383 be found between the patterns of ReHo, CV-ReHo, hrf-LE and med-LEig, with strong positive 384 weights for somatomotor network B $(\operatorname{SM}(B))$ and also control network $(C T R(C))$. Another interesting 385 pattern emerges for CV-BC, which displays both positive (CTR(A), VIS(B)) and negative weights 386 (TEMP/PAR, LIMBIC(A), SAL/VAN(A), DMN(B)).

387 Finally, the network-wise $\psi_{N j}$ values were correlated across predictors, to assess how similar the 388 RSNs were to one to another in terms of their SUVR-fMRI coupling (Figure 7c). When considering 389 only significant correlations ( $\mathrm{p}<0.05$, uncorrected), an interesting pattern emerges: some RSNs are 390 fairly isolated from the rest of the brain in their SUVR-fMRI association pattern (e.g. DMN(A), 391 DMN(C), VIS(A), VIS(B), SM(A), CTR(A)), with only 1-2 significant correlations with other RSNs; 392 other RSNs, instead, have many significant correlations, and thus are similar to many other networks 393 in their SUVR-fMRI coupling (SAL/VAN(A), DAN(A), DAN(B), CTR(B), CTR(C), DMN(B), 394 LIMBIC(B), SUB). 
Figure 7 - Multilevel SUVR modelling across networks - multivariable network-level estimates. Individual network parameter estimates $\left(\psi_{N j}\right.$, sum of fixed effects $\theta_{N}$ and random effects $\eta_{N j}$, which describe the variability from the fixed effect for each $\operatorname{RSN} j$ ), plotted on the brain surface for each predictor (a). Correlation matrix (non-significant values in white, $p=0.05$, uncorrected) of the nine predictors' random effects $\eta_{N j}$ across RSNs (b). Correlation matrix (non-significant values in white, $\mathrm{p}=0.05$, uncorrected) of the RSNs' parameter estimates $\left(\psi_{N j}=\theta_{N}+\eta_{N j}\right)$ across predictors $(\mathbf{c})$.

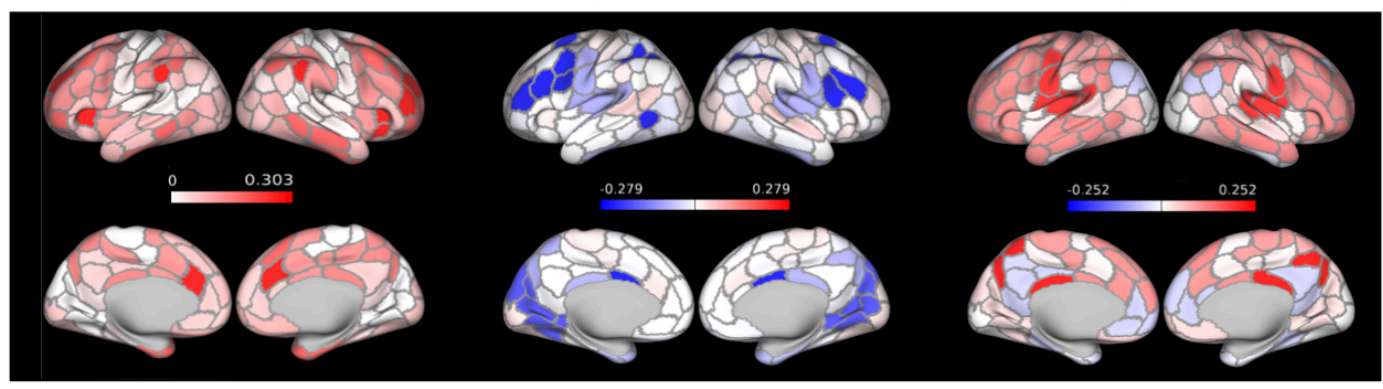

[4] CV-ReHo

[5] peaks-BOLD

[6] hrf-LE

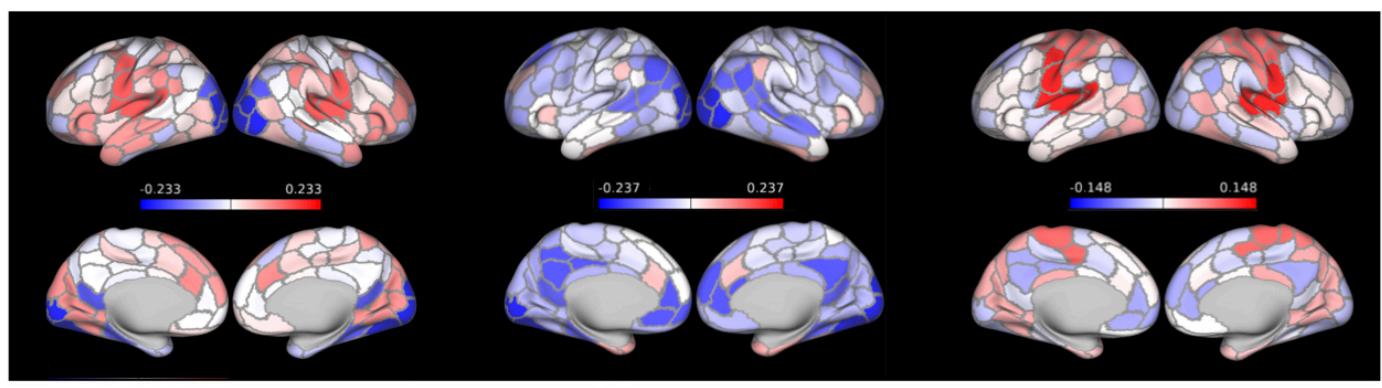

[7] s-BC

[8] CV-BC

[9] med-LEig

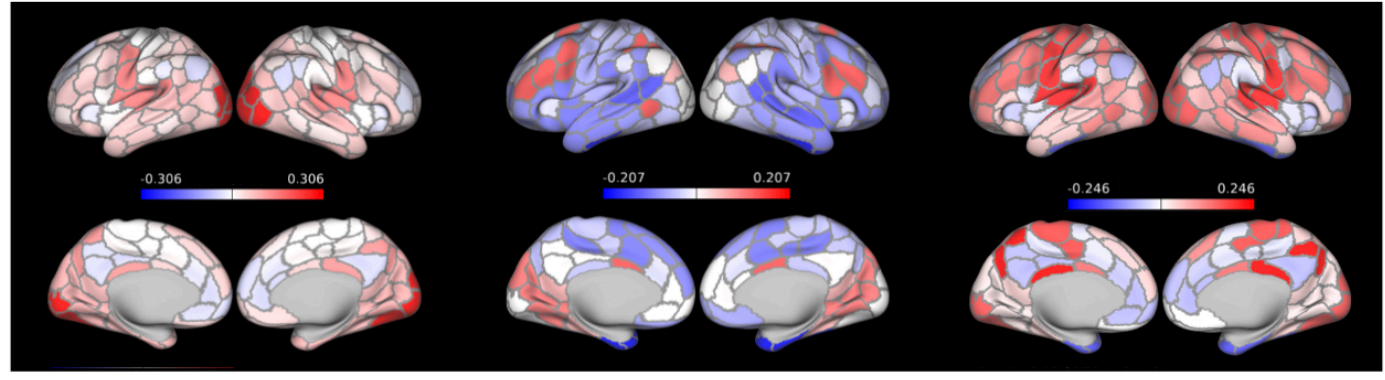

b Correlation of random effects across networks

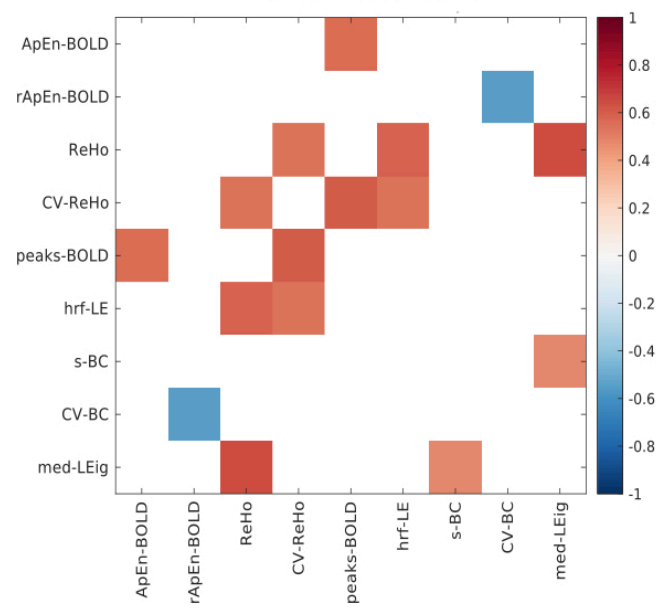

C Correlation of network-wise estimates across predictors

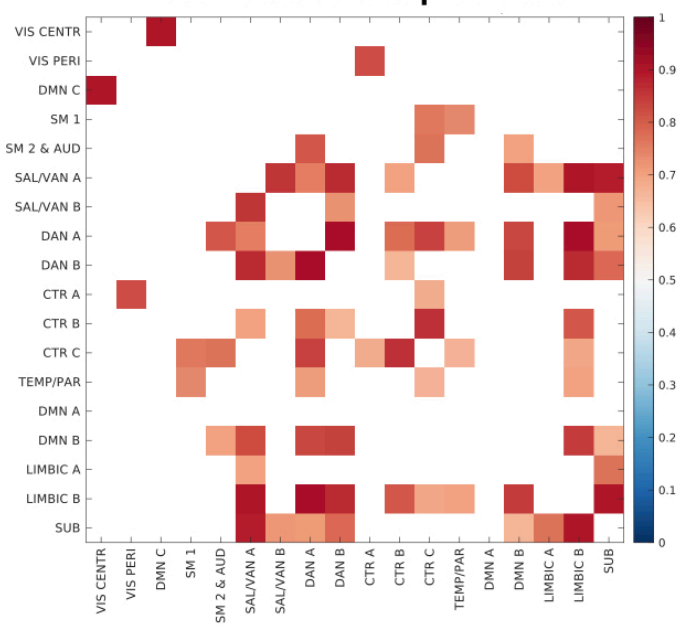


In this work, we thoroughly investigated the spatial relationship between a wide range of features extracted from rs-fMRI and simultaneously acquired $\left[{ }^{18} \mathrm{~F}\right]$ FDG PET, while also accounting for the variability across subjects (i.e., BSV) and networks (i.e., BNV) in this relationship.

\section{Relationship between SUVR and rs-fMRI through bivariate correlation}

410 In addition to the rs-fMRI variables that have already been associated to SUVR, i.e., ALFF, ReHo, 411 sFC strength ${ }^{22,23,25}$, we extended our assessment to a wide variety of previously unexplored features, 412 such as time-varying functional connectivity (tvFC) and HRF-related variables.

413 To our knowledge, the relationship between $\left[{ }^{18} \mathrm{~F}\right] \mathrm{FDG}$ metabolism and FC temporal variability has 414 never been tested before. While it is established that regions with stronger static FC tend to have 415 higher cerebral blood flow ${ }^{41}$ and higher energy metabolism ${ }^{25}$, possibly reflecting the fact that they are 416 also more strongly connected anatomically ${ }^{42}$ (with recent work highlighting that structural 417 connectivity graph properties are positively associated with $\left[{ }^{18} \mathrm{~F}\right] \mathrm{FDG}$ SUVR $\left.{ }^{43,44}\right)$, the tvFC coupling 418 with glucose metabolism is not established.

419 We found that tvFC (as captured by graph theory metrics' temporal variability) has a moderate-strong 420 negative association with SUVR. The interpretation of this finding can be supported by knowing that 421 sFC and tvFC graph metrics are negatively correlated, as clearly shown by the correlation matrix among rs-fMRI predictors (Figure 2a) and that the higher the strength of a static correlation across the entire rs-fMRI acquisition, the lower its temporal variability across time windows from the same acquisition ${ }^{45}$. However, examination of the relationships between $\mathrm{tvFC}$ and $\mathrm{sFC}$ graph metrics with SUVR suggests that a different pattern of correlations emerges, with tvFC-SUVR associations not being just the inverse of the sFC findings (Figure 2b). Similarly to our findings with $\left[{ }^{18} \mathrm{~F}\right] \mathrm{FDG}, \mathrm{tvFC}$ has been previously linked to L- $\left[1-{ }^{11} \mathrm{C}\right]$ leucine PET, with regions having strong protein turnover displaying less temporal variability of their graph properties ${ }^{46}$. Interestingly, this negative association stands out when compared to CV-ReHo, which is instead positively associated with SUVR, implying that the dynamics of local synchronization vs. global FC may have different neurobiological underpinnings: this is in line with a rs-fMRI study which showed that, unlike with FC, static ReHo and time-varying ReHo are positively correlated ${ }^{47}$. Additionally, a strong negative relationship is found between SUVR and the number of BOLD pseudo-events (peaks-BOLD), which is related to the interpretation of the BOLD signal as a point process, with sparse neural events governing its dynamics ${ }^{48}$. While puzzling at first, one interpretation might come from considering that higher local oxygen consumption by active neurons is associated with decreased positive BOLD fluctuations ${ }^{49}$, and therefore the higher the number of BOLD peaks and extreme events, the lower the oxidative metabolism and SUVR might be in that region. 
We then examined how these relationships would be modulated by selecting parcels according to their ranking in the SUVR distribution. In order to better probe the spatial relationship between SUVR and rs-fMRI, which is heterogeneous across the brain ${ }^{23,28}$, we chose to explore the changes in correlations selecting nodes from the SUVR standpoint, instead of according to FC properties ${ }^{24,25}$. Interestingly, nodes with progressively higher SUVR, which are expected to be the richest in terms of receptor density, local activity and inter-regional communications ${ }^{1,3,4}$, did not show different relationships between metabolism and rs-fMRI, but the correlations with most rs-fMRI features became significantly stronger when considering nodes with progressively lower SUVR (Figure 3). This finding suggests that only in nodes with lower metabolism is the $\left[{ }^{18} \mathrm{~F}\right] \mathrm{FDG}-\mathrm{fMRI}$ relationship emphasized, implying the presence of a nonlinear association for most of the rs-fMRI features, not just for the previously explored metrics ${ }^{25,28}$, with high SUVR nodes remaining unexplained by the available features. This nonlinear association was also assessed through model selection, with either an exponential, a power law or a log-linear relationship attributed to the majority $(86 \%)$ of the evaluated bivariate associations (see Supplementary Results). The nonlinearity of the coupling between glucose consumption and BOLD is partly expected: 1) known nonlinearity exists in the associations between BOLD and neuronal activity ${ }^{18}$, to which glucose metabolism is instead linearly related $^{7}$; 2) nonlinear models such as power laws are common in biology, and in particular in metabolic budget ${ }^{25}$; 3) the $\left[{ }^{18} \mathrm{~F}\right] \mathrm{FDG}$ coupling with local and global $\mathrm{FC}^{25,28}$ has been previously described with a power law within specific areas; 4) nonlinear relationships between cerebral blood flow and glucose metabolism have been reported ${ }^{50}$.

\section{The multivariable multilevel model}

461 To our knowledge, this is the first study to investigate the [ $\left.{ }^{18} \mathrm{~F}\right]$ FDG-fMRI coupling using a multivariable approach, attempting to identify the best subset of metrics, among a wide range of fMRI-derived variables, to explain SUVR variability across regions. Moreover, to fully capitalize on the fact that PET and fMRI data were acquired in the same subjects, we employed a multilevel modelling approach, with the selection of the best features performed at the group (higher) level, and modelling performed at the individual (lower) level, to characterize the between-subject variability of the SUVR-fMRI association (Figure 4, top). The selected model consisted of nine rs-fMRI variables (Figure 5 a, b) which represented all pools of features: signal (ApEn-BOLD, rApEnBOLD, peaks-BOLD, ReHo, CV-ReHo), HRF (hrf-LE), sFC (s-BC), tvFC (CV-BC), PC (med-Leig). The strongest predictors are related to the BOLD signal and its local synchronization properties (peaks-BOLD, ReHo), which consistently emerged as relevant across all feature selection methods (Supplementary Results). The fact that the SUVR-fMRI spatial coupling is emphasized when local 
473 BOLD variables are involved might reflect the interplay between excitatory and inhibitory neural 474 populations $^{51}$, which regulate $\mathrm{CBF}$, a main ingredient in many fMRI-related features ${ }^{17,18,52,53}$.

475 Overall, the explanatory power provided by BOLD rs-fMRI reached a $40 \%$ of the SUVR variance at 476 the group level (24\% across subjects). Zones of polarization in the model residuals emerged in 477 subcortical (putamen), posteromedial (PCC), and lateral frontal regions, which could mainly be 478 attributed to outliers with higher metabolism (Figure 5c), which are poorly explained by the available 479 rs-fMRI features in a consistent manner across subjects (Figure 5d). These results point to the idea 480 that the BOLD signal and $\mathrm{FC}$, even though related to $\mathrm{CBV}, \mathrm{CMRO}_{2}$ and $\mathrm{CBF}^{17,18}$, reflect the 481 metabolic architecture established by $\left[{ }^{18} \mathrm{~F}\right] \mathrm{FDG}$ SUVR only partially, even in simultaneously 482 acquisitions, and that rs-fMRI FC and its graph metrics cannot be considered a proxy of glucose 483 metabolism.

484 Moreover, the individual model $\mathrm{R}^{2}$ values were variable across subjects, highlighting the fact that the 485 SUVR-fMRI relationship displays significant between-subject variability, with subjects whose 486 BOLD signal and FC architecture are more related to SUVR, and others that have hardly any 487 relationship.

488 Next, we used the multilevel modelling approach and the identified predictors to characterize 489 between-network variability, exploiting the fMRI-derived RSNs to group the individual data in a 490 network-by-network fashion (Figure 4, bottom).

491 Importantly, the rs-fMRI predictors selected for the between-subject model proved to still be relevant 492 for evaluating the between-network SUVR-fMRI association, but their ranking, as assessed by 493 dominance analysis, changed noticeably (Figure 6b), with static and dynamic FC features (CV-BC 494 in particular) gaining importance in the model.

495 Moreover, when the network-wise effects are considered, significant positive and negative 496 associations emerge for each of the nine predictors (Figure 7a). These patterns of predictors have some degree of similarity across networks, with a cluster of RSNs (subcortical, limbic, salience, dorsal attention etc.) being highly correlated, which implies they have a similar SUVR-fMRI multivariable association pattern (Figure 7c). Other networks, instead, seem to be more isolated in their SUVR-fMRI coupling (default mode, visual, somatomotor etc.), possibly reflecting their 502 (Figure 5c).

503 These findings add to and enrich previous work highlighting between-network variability in the 504 SUVR-fMRI association through bivariate associations ${ }^{23,28}$.

505 Notably, the regions where SUVR-fMRI bivariate correlations are higher (Figure 3) seem to fall into 506 networks with fairly high $\mathrm{R}^{2}$ values in the multivariable model (Figure 6c), confirming that the 507 SUVR-fMRI coupling is emphasized in these regions. It is also important to underline that marked 
regional heterogeneity has also been described in the coupling between $\left[{ }^{18} \mathrm{~F}\right] \mathrm{FDG}$ SUVR and local

$509 \mathrm{CBF}^{54}$, which is likely to underlie some of the variability detected here due to CBF contributions to rs-fMRI variables.

\section{Limitations}

513 A comprehensive understanding of the relationship between $\left[{ }^{18} \mathrm{~F}\right] \mathrm{FDG}$ PET and rs-fMRI will require 514 simultaneous assessment of other features, such as $\mathrm{CBF}$ and $\mathrm{CMRO}_{2}$, ideally probed by $\left[{ }^{15} \mathrm{O}\right] \mathrm{H}_{2} \mathrm{O}$ $515 \mathrm{PET}^{55}$ and $\left[{ }^{15} \mathrm{O}\right] \mathrm{O}_{2} \quad \mathrm{PET}^{56}$ respectively, which would thoroughly describe the effects of 516 hemodynamics and oxidative and non-oxidative metabolism, possibly bridging the gap between these measures. Additionally, while the dataset employed here consists of standard rs-fMRI acquisitions (single-echo, TR of $2 \mathrm{~s}$, voxel size $3-4 \mathrm{~mm}$, duration $\sim 10$ minutes), the BOLD- $\left[{ }^{18} \mathrm{~F}\right] \mathrm{FDG}$ coupling would likely be improved by more advanced rs-fMRI methods (e.g. multi-echo imaging ${ }^{57}$ ), recordings of physiological variables (respiratory volume, end-tidal $\mathrm{CO}_{2}$ and heart rate) ${ }^{20}$, and regression of the CBF contribution ${ }^{52}$ out of the BOLD signal and local coherence features. For what concerns $\left[{ }^{18} \mathrm{~F}\right]$ FDG PET, it must also be remembered that SUVR, which was employed here as well as in all the literature on $\left[{ }^{18} \mathrm{~F}\right] \mathrm{FDG}-\mathrm{fMRI}$ coupling $22,23,25,27,28$, may offer a biologically confounded view of glucose consumption: SUVR is in fact a semi-quantitative and relative index, which results from interactions between the rate constants of the $\left[{ }^{18} \mathrm{~F}\right] \mathrm{FDG}$ compartmental model, i.e., $\mathrm{K}_{1}\left(\mathrm{ml} / \mathrm{cm}^{3} / \mathrm{min}\right)$, describing tracer uptake through the blood-brain barrier, $\mathrm{k}_{2}\left(\mathrm{~min}^{-1}\right)$, describing its efflux into the venous blood, and $\mathrm{k}_{3}\left(\mathrm{~min}^{-1}\right)$, quantifying the phosphorylation rate of the hexokinase in neurons and glia ${ }^{1,2}$. There is therefore the possibility that some of the contribution of CBF to the $\left[{ }^{18} \mathrm{~F}\right] \mathrm{FDG}-\mathrm{fMRI}$ coupling comes from SUVR, which is in fact highly correlated with the early, CBFrelated frames of $\left[{ }^{11} \mathrm{C}\right] \mathrm{PiB}$ PET in healthy controls ${ }^{59}$, as well as with PET-derived CBF estimates ${ }^{54}$. It is therefore likely that PET kinetic modelling will help disentangle the biological processes underlying both BOLD rs-fMRI and static PET estimates.

\section{Conclusion}

535 In conclusion, we thoroughly investigated for the first time the spatial relationship between $\left[{ }^{18} \mathrm{~F}\right] \mathrm{FDG}$ 536 SUVR and a wide range of features derived from rs-fMRI (pooled into 1) signal, 2) HRF, 3) sFC, 4) 537 tvFC and 5) PC-based features) using simultaneous PET/fMRI data. Selection of low SUVR parcels 538 led to a strengthening of SUVR-fMRI associations, implying the presence of a nonlinear relationship for many features. Moreover, a novel multivariable multilevel modelling framework was employed to identify the best subset of rs-fMRI predictors able to explain regional SUVR variance, highlighting that predictors based on the BOLD signal and its local synchronization (ReHo and BOLD pseudoevents, in particular) are the ones that are more tightly related to $\left[{ }^{18} \mathrm{~F}\right] \mathrm{FDG}$ SUVR across brain 
543 regions. This suggests a local contribution of CBF that should be tested for and, possibly, regressed 544 out from the BOLD signal.

545 Notably, the overall explanatory power provided by rs-fMRI on the regional metabolic variability did 546 not exceed $40 \%$ of the variance at the group level, with significant variability across subjects. When 547 multilevel modelling of the SUVR-fMRI coupling was carried out across networks, the selected 548 predictors were still relevant for description of RSN metabolism, but noticeable variability across 549 networks was present: new positive and negative associations emerged, and sFC and tvFC network 550 features gained importance. In conclusion, SUVR variability across parcels is only partly expression 551 of brain network organization described by rs-fMRI. 
555 ALFF, amplitude of low frequency fluctuations

556 ApEn, approximate entropy

$557 \mathrm{BC}$, betweenness centrality

558 BNV, between-network variability

559 BOLD, blood oxygen level dependent

560 BSV, between-subject variability

561 CBF, cerebral blood flow

$562 \mathrm{CBV}$, cerebral blood volume

$563 \mathrm{CMRO}_{2}$, cerebral metabolic rate of oxygen

564 CTR, control network

$565 \mathrm{CV}$, coefficient of variation

566 DAN, dorsal attention network

567 DMN, default mode network

568 FC, functional connectivity

569 FDG, fluorodeoxyglucose

570 FDR, false discovery rate

571 fMRI, functional magnetic resonance imaging

572 HRF, hemodynamic response function

573 LE, local efficiency

574 LIMBIC, limbic network

575 LME, linear mixed-effect

576 MAD, median absolute deviation

577 med-LEig, median of Leading Eigenvectors

578 OLS, ordinary least squares

579 NAD, naïve average data

580 NNLS, non-negative least squares

581 PC, phase coherence

582 PET, positron emission tomography

583 rApEn, range approximate entropy

584 ReHo, regional homogeneity

585 rs-fMRI, resting-state fMRI

586 RSN, resting-state network

587 SAL/VAN, salience/ventral attention network

588 SE, standard error 
589 sFC, static FC

590 SM, somatomotor network

591 STR, strength

592 SUB, subcortical regions

593 SUVR, standard uptake value ratio

594 TEMP/PAR, temporo-parietal network

595 tvFC, time-varying $\mathrm{FC}$

596 VIS, visual network

597

598

599

600 


\section{Data and Imaging protocols}

The dataset includes 26 healthy subjects from two studies: 11 subjects ( 8 males; $52.2 \pm 10.4$ years), hereby referred to as dataset A (Munich) ${ }^{23}$, and 15 subjects ( 6 males; $64.7 \pm 7.9$ years), i.e., dataset B (Naples) $^{31}$. Subjects were scanned in eyes open condition while looking at a fixation cross. Exclusion criteria included a history of psychiatric or neurological disorders, use of psychoactive drugs or treatment with CNS-active medications, pregnancy, and MR-related contraindications. The subjects provided their informed written consent according to the Code of Ethics of the World Medical Association and the Institutional Review Board and Ethics Committee at the Technische Universität München, for dataset A, and the SDN Foundation, for dataset B. Both centers simultaneously collected $\left[{ }^{18} \mathrm{~F}\right] \mathrm{FDG}$ PET and rs-fMRI data accompanied by a structural MR image on two identical Biograph mMR 3T scanners (Siemens Healthcare, Erlangen, Germany) equipped with the standard-supply head-neck coil (12-channel).

Dataset A: MRI data consisted in a structural magnetization prepared rapid acquisition gradient echo $\left(\right.$ MPRAGE) T1-weighted $(\mathrm{T} 1 \mathrm{w})$ image $\left(\mathrm{TR} / \mathrm{TE}=2300 / 2.98 \mathrm{~ms}, \mathrm{FA}=9^{\circ}, 1 \mathrm{~mm}\right.$ isotropic voxel size with $0.5 \mathrm{~mm}$ gap), 300 volumes of $\mathrm{T} 2 *$-weighted gradient-echo echo-planar imaging (GE-EPI) with $\mathrm{TR} / \mathrm{TE}=2000 / 30 \mathrm{~ms}$ and voxel size of $3 \mathrm{~mm}$ isotropic (0.6 mm inter-slice gap). PET acquisition consisted in a saturated list mode (10 min duration), started 30 minutes post-injection and reconstructed with voxel size of $3.7 \times 2.3 \times 2.7 \mathrm{~mm}^{3}$.

Dataset B: MRI data consisted in a similar T1- MPRAGE structural image and 240 volumes of GEEPI for rs-fMRI with $4 \mathrm{~mm}$ isotropic voxel and TR/TE $=1920 / 32 \mathrm{~ms}$. Simultaneous PET/fMRI measurements started 30 min post-injection, and PET images were acquired in sinogram mode for 15 min with reconstruction voxel size of $1.12 \times 1.12 \times 2.0 \mathrm{~mm}^{3}$. The interested reader should refer to the respective papers ${ }^{23,31}$ for more detailed information on each dataset.

\section{Data pre-processing}

631 All subjects were identically pre-processed to obtain local metabolism information from $\left[{ }^{18} \mathrm{~F}\right] \mathrm{FDG}$ 632 PET data, and BOLD-based measures from rs-fMRI data, employing a pipeline similar to the Human Connectome Project (HCP) minimal preprocessing pipeline ${ }^{60}$ with the addition of PET processing. 
636 Structural T1w images were N4 bias field-corrected ${ }^{61}$, skull-stripped, and segmented into grey matter 637 (GM), white matter (WM) and cerebrospinal fluid. The brain cortex was delineated with Freesurfer 638 (recon-all volume and surface reconstruction pipelines) ${ }^{62}$, obtaining pial and GM-WM interface 639 surfaces. Manual editing was performed to correct for surface delineation errors. Generated surfaces 640 were resampled over the $f_{S \_} L R$ mesh provided by Conte 69 atlas (symmetric-hemisphere mesh of $32 \mathrm{k}$ 641 nodes) to obtain aligned cortical surfaces for each subject. The Schaefer functional atlas ${ }^{32}$ was used 642 to parcellate cortical surfaces into 200 parcels, grouped according to Yeo's 17 RSNs scheme ${ }^{12}$ into 643 Central Visual (VIS(A)), Peripheral Visual (VIS(B)), Somatomotor A (SM(A)), Somatomotor B 644 (SM(B)), Temporal Parietal (TP), Dorsal Attention A (DAN(A)), Dorsal Attention B (DAN(B)), 645 Salience/Ventral Attention A (VAN(A)), Salience/Ventral Attention B (VAN(B)), Control A 646 (CTR(A)), Control B (CTR(B)), Control C (CTR(C)), Default Mode A (DMN(A)), Default Mode B 647 (DMN(B)), Default Mode C (DMN(C)), Limbic A (L(A)) and Limbic B (L(B). The cortical regions 648 were supplemented by 18 subcortical regions extracted from Freesurfer (bilaterally: Caudate, 649 Putamen, Accumbens, Pallidum, Amygdala, Hippocampus, Thalamus, Ventral diencephalon, 650 Cerebellar cortex) and delineated in single-subject space employing the Multi-Atlas Label Fusion 651 (MALF) method ${ }^{33}$. Parcels corresponding to subcortical regions were assigned to the Subcortical 652 (SUB) group.

PET data pre-processing

$655\left[{ }^{18} \mathrm{~F}\right]$ FDG PET images, after normalization to injected dose and subject's body weight, were linearly 656 resampled in T1w space with FSL's flirt $t^{63}$ and intensity-normalized by the whole-brain average 657 uptake 64 :

$S U V=\frac{\left[{ }^{18} \mathrm{~F}\right] \mathrm{FDG} \text { concentration }[\mathrm{MBq} / \mathrm{ml}]}{\frac{\text { injected dose }[\mathrm{MBq}]}{\text { body weight }[\mathrm{kg}]}} \quad S U V R=\frac{S U V_{\text {target }}}{S U V_{\text {reference }}}$

SUVR maps were then parcellated in the same way as the rs-fMRI data, and parcel-wise SUVR was computed as the median value of the vertices inside a region. All pre-processing steps avoided any further spatial smoothing on both $\left[{ }^{18} \mathrm{~F}\right] \mathrm{FDG}$ and rs-fMRI data.

\section{Functional MRI data pre-processing}

664 The first four rs-fMRI volumes were discarded to avoid non-equilibrium magnetization effects. The remaining volumes were corrected for slice timing difference by realigning them to the median volume, using FSL's mcflirt $^{63}$. A template EPI volume was obtained with antsBuildTemplate ${ }^{65}$ from realigned rs-fMRI data and used to estimate an affine transform (flirt, FSL), subsequently employed to map main tissue segmentations obtained from the pre-processed $\mathrm{T} 1 \mathrm{w}$ image to the native EPI 
space. Nuisance signals consisted in motion traces and their first order derivatives complemented by the first five temporal principal components, obtained after principal component analysis of WM and CSF EPI signals, explaining $70 \%$ and $50 \%$ of the average variance across subjects ${ }^{66}$, which were regressed out from all brain voxels in native EPI space ${ }^{67}$. Regression residuals were finally resampled first to the T1w space and then on top of the mid-thickness cortical surface mesh with Connectome Workbench ${ }^{68}$. Finally, the BOLD signal was high-pass filtered with a cut-off of $0.008 \mathrm{~Hz}$. No lowpass filter was applied, as the higher frequency components (0.1-0.25 Hz) of BOLD are likely to provide relevant neural information ${ }^{69}$. The vertex-wise BOLD signal was parcellated according to the Schaefer cortical atlas and the supplementary subcortical MALF parcels as previously described.

\section{rs-fMRI feature extraction}

Feature extraction as well as subsequent analyses were performed in MATLAB (ver. 2020a, The Mathworks, Natick, MA).

50 different features were extracted from the BOLD signal, either at the vertex or the parcel level.

The extracted features were chosen as descriptors of different aspects of the BOLD 1) signal, 2) HRF, 3) sFC, 4) tvFC, and 5) PC. A list of the features and their acronyms is reported in Table 1. A detailed description of the features and how they were extracted can be found in the Supplementary Methods. The extracted features were then employed first in a bivariate correlation analysis against SUVR, and then in a multivariable multilevel modelling procedure to verify how much SUVR variance could be explained across the whole brain at the group level, as well as accounting for individual level information.

\section{Bivariate analysis of the metabolism-fMRI relationship}

rs-fMRI features vs. glucose metabolism across all brain regions

The bivariate relationship between node-wise SUVR and rs-fMRI properties was assessed at the group level (naïve average data approach, NAD), employing the region-wise median values across subjects for SUVR and each of the 50 extracted features. The association between fMRI-derived features and metabolism across nodes was separately tested via Spearman's rank bivariate correlation (significance level 0.05, corrected for multiple comparisons using the Benjamini-Hochberg FDR $\left.\operatorname{approach}^{36}\right)$.

\section{rs-fMRI features vs. metabolism in specific clusters of nodes}

The spatial heterogeneity in the $\left[{ }^{18} \mathrm{~F}\right] \mathrm{FDG}$ PET-fMRI relationship, which has previously been reported $^{23,25}$, was probed by selecting clusters of nodes with increasingly high or increasingly low SUVR. The threshold level was determined by considering linearly increasing percentiles of the 
SUVR distribution over all nodes, in the range going from the $1^{\text {st }}$ to $85^{\text {th }}$ percentiles, with step 1 (from 218 up to 33 nodes); moreover, in the opposite direction, nodes were selected according to linearly decreasing percentiles of SUVR, from the $100^{\text {th }}$ to the $15^{\text {th }}$ percentile (from 218 down to 33 nodes). Selected nodes at every level are reported in the binary matrix in Supplementary Figure S1. For each threshold level, Spearman's correlation between SUVR and all fMRI-derived features was calculated across the selected nodes, and FDR-corrected for multiple comparisons across thresholds and rs-fMRI features (significance level 0.05) ) $^{36}$ The absolute values of Spearman's correlation were summed across the 50 fMRI variables for each percentile, to determine which threshold had the maximum correlation across features.

\section{Multivariable modelling of the SUVR-fMRI relationship at the group level}

The relationship between SUVR and each of the 50 rs-fMRI properties $\left(f M R I_{i p}\right.$, for $i=1, \ldots, 218$ regions, and $p=1, \ldots, 50)$ was then tested with four different bivariate models:

1) a linear model,

\section{Results).}

process led to choose the log-linear model for multiple regression modelling (see Supplementary

At the NAD level, a multiple linear regression approach was employed to verify how much of the group-wise SUVR variance could be explained by the linear combination of different fMRI-based features. The ordinary least squares (OLS) problem was formulated as follows:

$$
y=X \beta+\varepsilon
$$

where $y$ and $\varepsilon$ are $n \times 1$ vectors of the response/dependent variable (i.e., SUVR) and the model error, and $X \in \mathbb{R}^{n \times p}$ is the matrix of $p$ regressors (i.e., log-transformed rs-fMRI predictors), or design matrix. Before performing OLS regression, all predictors were z-scored, i.e., centered and scaled by their standard deviation (SD). The outcome variable, i.e., SUVR, was z-scored as well, so no model intercept needed to be estimated. The solution to the OLS problem was obtained as

$$
\hat{\beta}=\left(X^{T} X\right)^{-1} X^{T} y
$$


The model design matrix consisted of 50 parameters. The model was formulated as follows:

$$
S U V R_{i}=\beta_{1} \cdot \log f M R I_{i 1}+\beta_{2} \cdot \log f M R I_{i 2}+\cdots+\beta_{p} \cdot \log f M R I_{i p}+\varepsilon_{i}
$$

for each observation $i=1, \ldots, n$. The relationships amongst the predictors were evaluated by Spearman's correlation (Figure 2), to assess the presence of strong correlations (i.e., multicollinearity). Since high multicollinearity amongst predictors is known to result in lower precision, switched signs of the coefficients, and a lack of statistical significance of the multivariable model $^{35}$, the ill-conditioning of the design matrix was quantified using the condition number, i.e.,

$$
\kappa(X)=\frac{\sigma_{\max }(X)}{\sigma_{\min }(X)}
$$

with $\sigma_{\max }(X)$ and $\sigma_{\min }(X)$ as the highest and lowest singular values of $X$, respectively. As a rule of thumb, $\kappa(X)$ requires attention if higher than $30^{35}$.

The OLS fit was obtained with all the rs-fMRI variables and interpreted as the highest possible predictive power that could be extracted from the available features. However, it is well-known that, in the case of overparameterized linear models, OLS is generally not useful, as many CVs\%, (i.e., percent error variance divided by the absolute value of the parameter estimates) are too high (CVs\% $>100 \%$ ) and the model is not a posteriori identifiable, so it should be rejected ${ }^{37}$. As discussed, performing feature selection at the individual level would lead to unstable estimates, so we continued to work at the group (i.e., NAD) level.

Eleven feature selection strategies, namely non-negative least squares (NNLS), elastic net regression, hierarchical clustering, stepwise selection, and general-to-specific modelling in different combinations were tested and compared to identify the best group of features to explain SUVR variability at group level. More details are in (Supplementary Figure S4, Supplementary Table

\section{S1, Supplementary Methods).}

\section{Full hierarchical modelling of the SUVR-fMRI relationship}

As a NAD approach like the one described so far is statistically sound and unbiased only in case of low between-subject variability, a multilevel population modelling approach (mixed-effect model) was employed in order to characterize in a single stage both the group-level (fixed) and individuallevel (random) effects ${ }^{30}$ contributing to the relationship between the selected rs-fMRI variables and SUVR (Figure 4, top). First, the link between model and SUVR was described at individual level by the following equation:

$$
\begin{gathered}
y_{S i}=F_{S i}\left(X_{S i}, \psi_{S i}\right) \\
z_{S i}=y_{S i}+v_{S i}
\end{gathered}
$$

with $y_{S i}$ as the SUVR model prediction for the $i$ th subject $(i=1, \ldots, m)$, which is a function of $X_{S i}$ (the fixed-effects design matrix composed by the features extracted from the rs-fMRI data of 
subject $i$ ), and the parameters to be estimated for subject $i, \psi_{S i} ; z_{S i}$ is the vector of the measured SUVR data of subject $i$ and $v_{S i}$ is the within-subject variability, or residual unexplained variability, assumed to be normally distributed with zero mean and variance $\sigma_{i}^{2}$.

Second, at population level, $\psi_{S i}$ was described by a function combining population parameters (or fixed effects, $\theta_{S}$ ), and random variability of individual parameters around the population mean (or random effects, $\eta_{S i}$ ), according to the following assumptions:

$$
\begin{gathered}
\eta_{S i} \sim N\left(0, \Omega_{S}\right) \\
\psi_{S i}=\theta_{S}+\eta_{S i}
\end{gathered}
$$

where $\eta_{S i}$ is assumed to be Gaussian, with zero mean, independent across individuals and with covariance matrix $\Omega_{S}$ (another population parameter); as a consequence, $\psi_{S i}$ have a normal distribution as well. The matrix $\Omega_{S}$ was assumed to be full.

The intra-individual (first level) model structure was composed by the nine features selected with the NAD approach, here at single-subject level. Data normalization was performed within subjects via zscoring. The inter-individual model (second level) describing the BSV of the parameters was set according to the aforementioned assumptions.

This estimation requires solving the penalized least squares problem, i.e., the penalized weighted residual sum of squares (PWRSS),

$$
\operatorname{PWRSS}\left(\Omega_{\mathrm{S}}, \theta, y_{S i} \mid Z_{S i}\right)=\operatorname{WRSS}\left(\Omega_{\mathrm{S}}, \theta_{S}, y_{S i} \mid Z_{S i}\right)+\left\|y_{S i} \mid Z_{S i}\right\|^{2}
$$

with $Z_{S i}$ as the random-effects design matrix. This nonlinear optimization problem was solved using the restricted maximum likelihood (REML) estimation method ${ }^{70}$. The standard errors (SE) were calculated for each $\theta_{S}$ parameter estimate as the square root of the diagonal of their covariance matrix. The overall and subject-wise multilevel model $\mathrm{R}^{2}$ were also evaluated. The residual unexplained variability $v_{S i}$ was evaluated by calculating its median and variability $(\mathrm{CV} \%)$ across subjects.

The hierarchical modelling approach was also performed across networks $(N)$ in order to characterize between-network variability. RSNs were used as the grouping factor instead of subjects in a model formulated as follows:

$$
\begin{gathered}
y_{N j}=F_{N j}\left(X_{N j}, \psi_{N j}\right) \\
z_{N j}=y_{N j}+v_{N j}
\end{gathered}
$$

with $j$ as the $j$ th network $(j=1, \ldots, q)$. Normalization of SUVR and rs-fMRI variables was performed via z-scoring within RSNs.

The random effects $\eta_{N j}$ and the resulting individual parameters $\psi_{N j}$ were evaluated in terms of their correlation structure, both across RSNs ( $1^{\text {st }}$ dimension$)$ and across the nine predictors ( $2^{\text {nd }}$ dimension$)$, as seen in (Figure $\mathbf{7 b}, \mathbf{c})$. 
808 Relative importance analysis to determine predictor importance

809 Relative importance analysis ${ }^{40,71}$ was employed as a supplement to the results of hierarchical 810 modelling. This type of analysis allows to appropriately partition the model's explained variance 811 amongst multiple predictors when there is still significant multicollinearity, which makes typical 812 indicators of importance (e.g., standardized regression coefficients) flawed. Dominance analysis 813 (DA), in particular, works by rank-ordering the predictors in term of relative importance by 814 comparing the additional contributions they make to the $\mathrm{R}^{2}$ of all possible subset models. Specifically, 815 we assessed the general dominance of the variables, which is established for one predictor over 816 another when the average of its conditional contributions over all model sizes is greater than that of 817 the other. The obtained general dominance weights are also measures of relative effect sizes, as they 818 sum to the model $\mathrm{R}^{2}$ : the percent contribution to the model $\mathrm{R}^{2}$ was therefore calculated and reported. 819 While DA was originally proposed for OLS models, it was later extended to multilevel models ${ }^{40}$. In 820 order to apply DA to hierarchical models, a null model with no predictors must be provided, and the 821 slopes of first-level models must be considered fixed even when they are random in the identified 822 model, to simplify dominance evaluation. DA was used to assess the extent to which each selected 823 variable was driving the prediction in the context of the LME models across subjects $(S)$ and across 824 networks $(N)$, as they were still affected by non-negligible multicollinearity. 
830 The multimodal $\left[{ }^{18} \mathrm{~F}\right] \mathrm{FDG}$ PET and rs-fMRI data used in the present study can be accessed via 831 request to the groups who performed the original studies ${ }^{23,31}$. Data sharing will be subject to the 832 policies and procedures of the institution where each dataset was collected.

833 The codes and processed data that support the conclusions of this research work can be accessed via 834 request to the corresponding authors.

836 Acknowledgements:

837 The authors would like to thank Dr. Valentin Riedl for making dataset A available, Dr. Chiara 838 Favaretto for constructive suggestions for the analysis, and Dr. Mattia Veronese for his helpful and 839 insightful observations on the final version of the manuscript.

\section{Author contributions:}

842 T.V. and A.B. designed the study, performed the analysis, interpretation of the data, and the drafting 843 of the article. E.S. contributed to the preprocessing of the data and to the drafting of the article. M.C. 844 contributed to the interpretation of the data and to the drafting of the article. M.A. designed and 845 collected the data for the original study for dataset B and contributed to revision of the draft. All 846 authors approved the final version of the article to be published.

\section{Competing interests:}

849 The authors declare no competing interests. 


\section{References}

1. Sokoloff, L. et al. The $\left[{ }^{14} \mathrm{C}\right]$ deoxyglucose method for the measurement of local cerebral glucose utilization: theory, procedure, and normal values in the conscious and anesthetized albino rat. Journal of Neurochemistry 28, 897-916 (1977).

2. Bertoldo, A., Rizzo, G. \& Veronese, M. Deriving physiological information from PET images: From SUV to compartmental modelling. Clinical and Translational Imaging 2, 239251 (2014).

3. Clarke, D. D. \& Sokoloff, L. Circulation and energy metabolism in the brain. in Basic Neurochemistry: Molecular, Cellular and Medical Aspects. 6th edition. Philadelphia: Lippincott-Raven vol. 81 (1999).

4. Sokoloff, L. et al. The effect of mental arithmetic on cerebral circulation and metabolism. Journal of Clinical Investigation 34, 1101-1108 (1955).

5. Raichle, M. E. The Brain's Dark Energy. Science 314, 1249-1250 (2006).

6. Magistretti, P. J. \& Allaman, I. A Cellular Perspective on Brain Energy Metabolism and Functional Imaging. Neuron 86, 883-901 (2015).

7. Sokoloff, L. Energetics of Functional Activation in Neural Tissues. Neurochemical Research 24, 321-329 (1999).

8. Biswal, B., Yetkin, F. Z., Haughton, V. M. \& Hyde, J. S. Functional Connectivity in the Motor Cortex of Resting Human Brain Using Echo-Planar MRI. Magnetic Resonance in Medicine 34, 537-541 (1995).

9. Fox, M. D. \& Raichle, M. E. Spontaneous fluctuations in brain activity observed with functional magnetic resonance imaging. Nat Rev Neurosci 8, 700-711 (2007).

10. Raichle, M. E. The restless brain: how intrinsic activity organizes brain function. Phil. Trans. R. Soc. B 370, 20140172 (2015).

11. Cole, M. W., Bassett, D. S., Power, J. D., Braver, T. S. \& Petersen, S. E. Intrinsic and taskevoked network architectures of the human brain. Neuron 83, 238-251 (2014). 
12. Thomas Yeo, B. T. et al. The organization of the human cerebral cortex estimated by intrinsic functional connectivity. Journal of Neurophysiology 106, 1125-1165 (2011).

13. Allen, E. A. et al. Tracking whole-brain connectivity dynamics in the resting state. Cerebral Cortex 24, 663-676 (2014).

14. Power, J. D. et al. Functional Network Organization of the Human Brain. Neuron 72, 665678 (2011).

15. van den Heuvel, M. P. \& Sporns, O. Network hubs in the human brain. Trends in Cognitive Sciences 17, 683-696 (2013).

16. Bullmore, E. \& Sporns, O. The economy of brain network organization. Nature Reviews Neuroscience 13, 336-349 (2012).

17. Shen, Q., Ren, H. \& Duong, T. Q. CBF, BOLD, CBV, and CMRO2 fMRIrs-fMRI signal temporal dynamics at 500-msec resolution. J. Magn. Reson. Imaging 27, 599-606 (2008).

18. Kim, S.-G. \& Ogawa, S. Biophysical and Physiological Origins of Blood Oxygenation LevelDependent fMRI Signals. J Cereb Blood Flow Metab 32, 1188-1206 (2012).

19. Buxton, R. B. \& Frank, L. R. A Model for the Coupling between Cerebral Blood Flow and Oxygen Metabolism during Neural Stimulation. J Cereb Blood Flow Metab 17, 64-72 (1997).

20. Chen, J. E. et al. Resting-state "physiological networks". NeuroImage 213, (2020).

21. Cecchin, D. et al. Brain PET and functional MRI: Why simultaneously using hybrid PET/MR systems? Quarterly Journal of Nuclear Medicine and Molecular Imaging 61, 345-359 (2017).

22. Nugent, A. C., Martinez, A., D’Alfonso, A., Zarate, C. A. \& Theodore, W. H. The Relationship between Glucose Metabolism, Resting-State fMRI BOLD Signal, and GABA A -Binding Potential: A Preliminary Study in Healthy Subjects and Those with Temporal Lobe Epilepsy. J Cereb Blood Flow Metab 35, 583-591 (2015).

23. Aiello, M. et al. Relationship between simultaneously acquired resting-state regional cerebral glucose metabolism and functional MRI: A PET/MR hybrid scanner study. NeuroImage 113, $111-121(2015)$. 
24. Marchitelli, R. et al. Simultaneous resting-state FDG-PET/fMRI in Alzheimer Disease: Relationship between glucose metabolism and intrinsic activity. NeuroImage 176, 246-258 (2018).

25. Tomasi, D., Wang, G. J. \& Volkow, N. D. Energetic cost of brain functional connectivity. Proceedings of the National Academy of Sciences of the United States of America 110, 1364213647 (2013).

26. Bernier, M., Croteau, E., Castellano, C. A., Cunnane, S. C. \& Whittingstall, K. Spatial distribution of resting-state BOLD regional homogeneity as a predictor of brain glucose

27. Wang, J. et al. The Relationship Among Glucose Metabolism, Cerebral Blood Flow, and Functional Activity: a Hybrid PET/fMRI Study. Mol Neurobiol (2021).

28. Shokri-Kojori, E. et al. Correspondence between cerebral glucose metabolism and BOLD reveals relative power and cost in human brain. Nat Commun 10, 690 (2019).

29. Cabral, J. et al. Cognitive performance in healthy older adults relates to spontaneous switching between states of functional connectivity during rest. Scientific Reports 7, (2017).

30. Hox, J. J., Moerbeek, M. \& Schoot, R. van de. Multilevel analysis: techniques and applications. Routledge (2017).

31. Riedl, V. et al. Local Activity Determines Functional Connectivity in the Resting Human Brain: A Simultaneous FDG-PET/fMRI Study. Journal of Neuroscience 34, 6260-6266 (2014).

32. Schaefer, A. et al. Local-Global Parcellation of the Human Cerebral Cortex from Intrinsic Functional Connectivity MRI. Cerebral Cortex 28, 3095-3114 (2018).

33. Wang, H. \& Yushkevich, P. A. Multi-atlas segmentation with joint label fusion and corrective learning — an open source implementation. Front. Neuroinform. 7, (2013).

34. Wu, G.-R. et al. A blind deconvolution approach to recover effective connectivity brain networks from resting state fMRI data. Medical Image Analysis 17, 365-374 (2013). 
35. Belsley, D. A. Conditioning diagnostics: collinearity and weak data in regression. (Wiley, 1991).

36. Benjamini, Y. \& Hochberg, Y. Controlling the False Discovery Rate: A Practical and Powerful Approach to Multiple Testing. Journal of the Royal Statistical Society: Series B (Methodological) 57, 289-300 (1995).

37. Cobelli, C., Foster, D. \& Toffolo, G. Identifiability of the tracer model. in Tracer Kinetics in Biomedical Research 109-163.

38. Meinshausen, N. Sign-constrained least squares estimation for high-dimensional regression. Electronic Journal of Statistics 7, 1607-1631 (2013).

39. Zou, H. \& Hastie, T. Regularization and variable selection via the elastic net. $J$ Royal Statistical Soc B 67, 301-320 (2005).

40. Luo, W. \& Azen, R. Determining Predictor Importance in Hierarchical Linear Models Using Dominance Analysis. Journal of Educational and Behavioral Statistics 38, 3-31 (2013).

41. Liang, X., Zou, Q., He, Y. \& Yang, Y. Coupling of functional connectivity and regional cerebral blood flow reveals a physiological basis for network hubs of the human brain. Proceedings of the National Academy of Sciences 110, 1929-1934 (2013).

42. Honey, C. J. et al. Predicting human resting-state functional connectivity from structural connectivity. PNAS 106, 2035-2040 (2009).

43. Bi, Q. et al. Relationship between the disrupted topological efficiency of the structural brain connectome and glucose hypometabolism in normal aging. NeuroImage 226, (2021).

44. Chen, Y., Lin, Q., Liao, X., Zhou, C. \& He, Y. Association of aerobic glycolysis with the structural connectome reveals a benefit-risk balancing mechanism in the human brain. Proc Natl Acad Sci USA 118, e2013232118 (2021).

45. Thompson, W. H. \& Fransson, P. The mean-variance relationship reveals two possible strategies for dynamic brain connectivity analysis in fMRI. Front. Hum. Neurosci. 9, (2015). 
46. Hellyer, P. J. et al. Protein synthesis is associated with high-speed dynamics and broad-band stability of functional hubs in the brain. NeuroImage 155, 209-216 (2017).

47. Deng, L., Sun, J., Cheng, L. \& Tong, S. Characterizing dynamic local functional connectivity in the human brain. Scientific Reports 6, (2016).

48. Zhang, X., Pan, W.-J. \& Keilholz, S. D. The relationship between BOLD and neural activity arises from temporally sparse events. NeuroImage 207, 116390 (2020).

49. Howarth, C., Mishra, A. \& Hall, C. N. More than just summed neuronal activity: how multiple cell types shape the BOLD response. Phil. Trans. R. Soc. B 376, 20190630 (2021).

50. Henriksen, O. M. et al. Interindividual and regional relationship between cerebral blood flow and glucose metabolism in the resting brain. J Appl Physiol (1985) 125, 1080-1089 (2018).

51. Muthukumaraswamy, S. D., Evans, C. J., Edden, R. A. E., Wise, R. G. \& Singh, K. D. Individual variability in the shape and amplitude of the BOLD-HRF correlates with endogenous GABAergic inhibition. Hum. Brain Mapp. 33, 455-465 (2012).

52. Li, Z., Zhu, Y., Childress, A. R., Detre, J. A. \& Wang, Z. Relations between BOLD fMRIDerived Resting Brain Activity and Cerebral Blood Flow. PLoS ONE 7, e44556 (2012).

53. Tong, Y. et al. Perfusion information extracted from resting state functional magnetic resonance imaging. J Cereb Blood Flow Metab 37, 564-576 (2017).

54. Huisman, M. C. et al. Cerebral blood flow and glucose metabolism in healthy volunteers measured using a high-resolution PET scanner. EJNMMI Res 2, 63 (2012).

55. Fan, A. P., Jahanian, H., Holdsworth, S. J. \& Zaharchuk, G. Comparison of cerebral blood flow measurement with [15 O]-water positron emission tomography and arterial spin labeling magnetic resonance imaging: A systematic review. Journal of Cerebral Blood Flow and Metabolism 36, 842-861 (2015).

56. Fan, A. P. et al. Quantification of brain oxygen extraction and metabolism with [15O]-gas PET: A technical review in the era of PET/MRI. NeuroImage 220, (2020). 
57. Kundu, P. et al. Multi-echo fMRI: A review of applications in fMRI denoising and analysis of BOLD signals. NeuroImage 154, 59-80 (2017).

58. Hamberg, L. M. et al. The dose uptake ratio as an index of glucose metabolism: useful parameter or oversimplification? J Nucl Med 35, 1308-1312 (1994).

59. Peretti, D. E. et al. Relative cerebral flow from dynamic PIB scans as an alternative for FDG scans in Alzheimer's disease PET studies. PLoS ONE 14, e0211000 (2019).

60. Glasser, M. F. et al. The Human Connectome Project's neuroimaging approach. Nat Neurosci 19, 1175-1187 (2016).

61. Tustison, N. J. et al. N4ITK: Improved N3 Bias Correction. IEEE Trans. Med. Imaging 29, $1310-1320$ (2010).

62. Fischl, B., Sereno, M. I. \& Dale, A. M. Cortical Surface-Based Analysis II: Inflation, Flattening, and a Surface-Based Coordinate System. http://www.idealibrary.com (1999).

63. Jenkinson, M., Beckmann, C. F., Behrens, T. E. J., Woolrich, M. W. \& Smith, S. M. FSL. NeuroImage 62, 782-790 (2012).

64. Byrnes, K. R. et al. FDG-PET imaging in mild traumatic brain injury: A critical review. Frontiers in Neuroenergetics 6, (2014).

65. Avants, B. B. et al. A reproducible evaluation of ANTs similarity metric performance in brain image registration. NeuroImage 54, 2033-2044 (2011).

66. Behzadi, Y., Restom, K., Liau, J. \& Liu, T. T. A component based noise correction method (CompCor) for BOLD and perfusion based fMRI. NeuroImage 37, 90-101 (2007).

67. Ciric, R. et al. Benchmarking of participant-level confound regression strategies for the control of motion artifact in studies of functional connectivity. NeuroImage 154, 174-187 (2017).

68. Marcus, D. S. et al. Informatics and Data Mining Tools and Strategies for the Human Connectome Project. Front. Neuroinform. 5, (2011). 
1004 69. Chen, J. E. \& Glover, G. H. BOLD fractional contribution to resting-state functional

1005 connectivity above $0.1 \mathrm{~Hz}$. NeuroImage 107, 207-218 (2015).

1006 70. Laird, N. M. \& Ware, J. H. Random-effects models for longitudinal data. Biometrics 38 , 1007 963-974 (1982).

1008 71. Tonidandel, S. \& LeBreton, J. M. Relative Importance Analysis: A Useful Supplement to 1009 Regression Analysis. J Bus Psychol 26, 1-9 (2011). 


\section{Supplementary Files}

This is a list of supplementary files associated with this preprint. Click to download.

- ManuscriptVolpietalNatCommunSupplementary.docx 